\title{
The VIMOS VLT Deep Survey ${ }^{\star} \star \star$
}

\section{Evolution of the non-linear galaxy bias up to $z=1.5$}

C. Marinoni ${ }^{1,2}$, O. Le Fèvre ${ }^{2}$, B. Meneux ${ }^{2}$, A. Iovino ${ }^{1}$, A. Pollo ${ }^{3}$, O. Ilbert², ${ }^{2}$ G. Zamorani ${ }^{5}$, L. Guzzo ${ }^{3}$, A. Mazure ${ }^{2}$, R. Scaramella ${ }^{4}$, A. Cappi ${ }^{5}$, H. J. McCracken ${ }^{6}$, D. Bottini ${ }^{7}$, B. Garilli ${ }^{7}$, V. Le Brun ${ }^{2}$, D. Maccagni ${ }^{7}$, J. P. Picat ${ }^{8}$, M. Scodeggio ${ }^{7}$, L. Tresse ${ }^{2}$, G. Vettolani ${ }^{9}$, A. Zanichelli ${ }^{9}$, C. Adami ${ }^{2}$, S. Arnouts ${ }^{2}$, S. Bardelli ${ }^{5}$, J. Blaizot $^{2}$, M. Bolzonella ${ }^{10}$, S. Charlot ${ }^{6,11}$, P. Ciliegi ${ }^{9}$, T. Contini ${ }^{8}$, S. Foucaud $^{7}$, P. Franzetti ${ }^{7}$, I. Gavignaud ${ }^{8}$, B. Marano ${ }^{10}$,

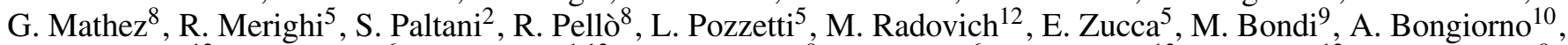
G. Busarello ${ }^{12}$, S. Colombi ${ }^{6}$, O. Cucciati ${ }^{1,13}$, F. Lamareille ${ }^{8}$, Y. Mellier ${ }^{6}$, P. Merluzzi $^{12}$, V. Ripepi ${ }^{12}$, and D. Rizzo ${ }^{8}$

1 INAF - Osservatorio Astronomico di Brera, via Brera 28, 20121 Milano, Italia

2 Laboratoire d'Astrophysique de Marseille, UMR 6110 CNRS-Université de Provence, Traverse du Siphon-Les trois Lucs, 13012 Marseille, France

e-mail: christian.marinoni@cpt.univ-mrs.fr

3 INAF - Osservatorio Astronomico di Brera, via Bianchi 46, 23807 Merate, Italia

4 INAF - Osservatorio Astronomico di Roma, via Osservatorio 2, 00040 Monteporzio Catone (Roma), Italia

5 INAF - Osservatorio Astronomico di Bologna, via Ranzani 1, 40127 Bologna, Italia

${ }^{6}$ Institut d'Astrophysique de Paris, UMR 7095, 98 bis Bd Arago, 75014 Paris, France

7 INAF - IASF, Via Bassini 15, 20133 Milano, Italia

${ }^{8}$ Laboratoire d'Astrophysique - Observatoire Midi-Pyrénées, Toulouse, France

9 INAF - Istituto di Radio-Astronomia, Via Gobetti 101, 40129 Bologna, Italia

${ }^{10}$ Università di Bologna, Dipartimento di Astronomia, via Ranzani 1, 40127 Bologna, Italia

11 Max Planck Institut fur Astrophysik, 85741 Garching, Germany

12 INAF - Osservatorio Astronomico di Capodimonte, via Moiariello 16, 80131 Napoli, Italia

13 Università di Milano-Bicocca, Dipartimento di Fisica, Piazza della scienza 3, 20126 Milano, Italia

Received 2 March 2005 / Accepted 17 June 2005

\section{ABSTRACT}

We present the first measurements of the Probability Distribution Function (PDF) of galaxy fluctuations in the four-passes, first-epoch VIMOSVLT Deep Survey (VVDS) cone, covering $0.4 \times 0.4$ deg between $0.4<z<1.5$. We show that the PDF of density contrasts of the VVDS galaxies is an unbiased tracer of the underlying parent distribution up to redshift $z=1.5$, on scales $R=8$ and $10 h^{-1} \mathrm{Mpc}$. The second moment of the PDF, i.e., the rms fluctuations of the galaxy density field, is to a good approximation constant over the full redshift baseline investigated: we find that, in redshift space, $\sigma_{8}$ for galaxies brighter than $\mathcal{M}_{B}^{c}=-20+5 \log h$ has a mean value of $0.94 \pm 0.07$ in the redshift interval $0.7<z<1.5$. The third moment, i.e., the skewness, increases with cosmic time: we find that the probability of having underdense regions is greater at $z \sim 0.7$ than it was at $z \sim 1.5$. By comparing the PDF of galaxy density contrasts with the theoretically predicted PDF of mass fluctuations we infer the redshift-, density- and scale-dependence of the biasing function $b(z, \delta, R)$ between galaxy and matter overdensities up to redshift $z=1.5$. Our results can be summarized as follows: i) the galaxy bias is an increasing function of redshift: evolution is marginal up to $z \sim 0.8$ and more pronounced for $z \gtrsim 0.8$; ii) the formation of bright galaxies is inhibited below a characteristic mass-overdensity threshold whose amplitude increases with redshift and luminosity; iii) the biasing function is non linear in all the redshift bins investigated with non-linear effects of the order of a few to $\sim 10 \%$ on scales $>5 h^{-1} \mathrm{Mpc}$. By subdividing the sample according to galaxy luminosity and colors, we also show that: iv) brighter galaxies are more strongly biased than less luminous ones at every redshift and the dependence of biasing on luminosity at $z \sim 0.8$ is in good agreement with what is observed in the local Universe; v) red objects are systematically more biased than blue objects at all cosmic epochs investigated, but the relative bias between red and blue objects is constant as a function of redshift in the interval $0.7<z<1.5$, and its value $\left(b^{\text {rel }} \sim 1.4\right)$ is similar to what is found at $z \sim 0$.

Key words. cosmology: large-scale structure of Universe - galaxies: distances and redshifts - galaxies: evolution galaxies: statistics

\footnotetext{
* Based on data obtained with the European Southern Observatory

Very Large Telescope, Paranal, Chile.

$\star \star$ Appendix A is only available in electronic form at

http://www.edpsciences.org
} 


\section{Introduction}

The understanding of how matter structures grow via gravitational instability in an expanding Universe is quite well developed and has led to a successful and predictive theoretical framework (e.g., Peebles 1980; Davis et al. 1985).

One of the most critical problems, however, is to understand the complex mechanisms which, on various cosmological scales, regulate the formation and the evolution of luminous structures within the underlying dark-matter distribution. Its solution ultimately relies on the comprehension of the "microscopic" physics which describes how the baryons fall, heat-up, virialize, cool and form stars in the potential wells generated by the dominant mass component of the Universe, i.e., the nonbaryonic dark matter (e.g., White \& Rees 1978). A zero-order, minimal approach to this investigation consists of "macroscopically" characterizing the cosmological matter fluctuations in terms of a reduced set of fundamental quantities, essentially their positions and mass scales, and in studying how the respective spatial clustering and density amplitudes relate to the corresponding statistics computed for light fluctuations. This comparison scheme is generally referred to as matter-galaxy biasing (e.g., Dekel \& Lahav 1999).

An operational definition of bias is conventionally given in terms of continuous density fields by assuming that the local density fluctuation pattern traced by galaxies $\left(\delta_{\mathrm{g}}\right)$ and mass $(\delta)$ are deterministically related via the "linear biasing scheme"

$\delta_{\mathrm{g}}(\delta)=b \delta$

where the constant "slope" $b$ is the biasing parameter (Kaiser 1984).

This specific formulation, however, represents a very crude approximation which is not based on any theoretical or physical motivation. It is obvious, for example, that such a model cannot satisfy the physical requirement $\delta_{\mathrm{g}}(-1)=-1$ for any arbitrary value $b \neq 1$. In particular the biasing process could be non local (e.g., Catelan et al. 1998), stochastic (e.g., Dekel \& Lahav 1999) and non linear (e.g., Mo \& White 1996). Moreover, both theory and numerical simulations predict that the bias grows monotonically from the present cosmic epoch to high redshifts (e.g., Dekel \& Rees 1987; Fry 1996; Mo \& White 1996; Tegmark \& Peebles 1998; Basilakos \& Plionis 2001).

From a theoretical perspective, light does not follow the matter distribution on sub-galactic scales, where nearly $90 \%$ of dense, low-mass dark matter fluctuations $\left(M \sim 10^{7}-10^{8} h^{-1} M_{\odot}\right)$ failed to form stars and to become galaxies (e.g., Klyplin et al. 1999; Moore et al. 1999; Dalal $\&$ Kochanek 2002). A difference in the spatial distribution of visible and dark matter is predicted also on galactic scales, since the radial scaling of density profiles of dark matter halos (Navarro et al. 1997) differs from the three-dimensional radial distributions of light (Sersic and Freeman laws). Galaxy biasing is theoretically expected also on cosmological scales. In particular, simulations of the large-scale structure predict the existence of a difference in the relative distribution of mass and dark halos (e.g., Cen \& Ostriker 1992; Bagla 1998; Kravtsov \& Klypin 1999) or galaxies (e.g., Evrard, et al. 1994; Blanton et al. 2000; Kayo et al. 2001). Various physical mechanisms for biasing have been proposed, such as, for example, the peaksbiasing scheme (Kaiser 1984; Bardeen et al. 1986), the probabilistic biasing approach (Coles 1993), or the biasing models derived in the context of the extended Press \& Schechter approximation (Mo \& White 1996; Matarrese et al. 1998).

Turning to the observational side, the fact that, in the local Universe, galaxies cluster differently according to morphological type (Davis \& Geller 1976), surface brightness (Davis \& Djorgovski 1985), luminosity (Maurogordato \& Lachièze-Rey 1987), or internal dynamics (White et al. 1988) implies that not all can simultaneously trace the underlying distribution of mass, and that galaxy biasing not only exists, but might also be sensitive to various physical processes. Redshift information that recently became available for large samples of galaxies has significantly contributed to better shaping our current understanding of galaxy biasing, at least in the local Universe. The analysis of the power spectrum (Lahav et al. 2002) and bispectrum (Verde et al. 2002) of the 2dF Galaxy Redshift Survey (2dFGRS Colless et al. 2001) consistently shows that a fluxlimited sample of local galaxies $(z<0.25)$, optically selected in the $b_{J}$-band $\left(b_{J} \leq 19.4\right)$, traces the mass, i.e., it is unbiased, on scales $5<R\left(h^{-1} \mathrm{Mpc}\right)<30$.

The galaxy correlation function has been measured up to redshift $\sim 1$ by the CFRS (Le Fèvre et al. 1996), and by the CNOC (Carlberg et al. 2000) surveys giving conflicting evidence on clustering amplitude and bias evolution (see Small et al. 1999). More recently, the analysis of the first season DEEP2 data (Coil et al. 2004) seems to indicate that a combined $R$-band plus color selected sample is unbiased at $z \sim 1$. On the contrary, measurements of the clustering (Steidel et al. 1998; Giavalisco . 1998; Foucaud et al. 2003) or of the amplitude of the count-in-cell fluctuations (Aldeberger et al. 1998) of Lyman-break galaxies (LBGs) at $z \sim 3$ suggest that these objects are more highly biased tracers of the mass density field than are galaxies today. Higher redshift domains have been probed by using photometric redshift information (Arnouts et al. 1999), or compilation of heterogeneous samples (Magliocchetti et al. 2000). Again, the clustering signal appears to come from objects which are highly biased with respect to the underlying distribution of mass.

While there is general observational consensus on the broad picture, i.e., that biasing must decrease with cosmic time, the elucidation of the finer details of this evolution as well as any meaningful comparison with specific theoretical predictions is still far from being secured. Since clustering depends on morphology, color and luminosity, and since most high redshift samples have been selected according to different colors or luminosity criteria, it is not clear, for example, how the very different classes of objects (Ly-break galaxies, extremely red objects or ultraluminous galaxies), which populate different redshift intervals, can be considered a uniform set of mass tracers across different cosmic epochs. Furthermore, the biasing relation is likely to be nontrivial, i.e., non-linear and scale dependent, especially at high redshift (e.g., Somerville et al. 2001).

Only large redshift surveys defined in terms of uniform selection criteria and sampling typical galaxies (or their progenitors), rather than small subclasses of peculiar objects, promise to yield a more coherent picture of biasing evolution. 
In particular, the 3D spatial information provided by the VIMOS-VLT Deep Survey (VVDS, Le Fèvre et al. 2005a, hereafter Paper I) should allow us to investigate the mass and scale dependence, as well as to explore the time evolution of the biasing relation between dark matter and galaxies for a homogeneous, flux-limited $(I \leq 24)$ sample of optically selected galaxies.

The intent of this paper is to provide a measure, on some characteristic scales $R$, over the continuous redshift interval $0.4<z<1.5$, of the local, non-linear, deterministic biasing function

$b=b(z, \delta, R)$.

The goal is to provide an observational benchmark for theories predicting the efficiency of structure formation, or semianalytical simulations of galaxy evolution. The problem, however, is to find an optimal strategy to evaluate the biasing function in the quasi-pencil-beam geometry of the first-epoch VVDS survey. At present, the angular size of the first-epoch VVDS redshift cone $\left(\sim 0.5 \mathrm{deg}^{2}\right)$ does not allow us to constrain the biasing function using high order moments of the galaxy distribution (for example the 3-point correlation function). Moreover, we cannot determine the biasing function simply regressing the galaxy fluctuations $\left(\delta_{\mathrm{g}}\right)$ versus mass fluctuations $(\delta)$. The fundamental limitation preventing such an intuitive comparison is evident: it is easy to "pixelize" the survey volume and to measure the galaxy fluctuations in each survey cell, but, since the VVDS is not a matter survey, it is much less straightforward to assign to each cell a mass density value. Thus, in this paper, we take an orthogonal approach and we infer the biasing relation $\delta_{\mathrm{g}}=\delta_{\mathrm{g}}(\delta)$ between mass and galaxy overdensities from their respective probability distribution functions (PDFs) $f(\delta)$ and $g\left(\delta_{\mathrm{g}}\right.$ ): assuming a one-to-one mapping between mass and galaxy overdensity fields, conservation of probability implies

$\frac{\mathrm{d} \delta_{\mathrm{g}}(\delta)}{\mathrm{d} \delta}=\frac{f(\delta)}{g\left(\delta_{\mathrm{g}}\right)}$.

The advantage over other methods is that we can explore the functional form of the relationship $\delta_{\mathrm{g}}=b(z, \delta, R) \delta$ over a wide range in mass density contrasts, redshift intervals and smoothing scales $R$ without specifying any a priori parametric functional form for the biasing function.

In pursuing our approach, we assume that the PDF of matter overdensities $f(\delta)$ is satisfactorily described by theory and $\mathrm{N}$-body simulations. What we will try to assess explicitly, is the degree at which the measured PDF of the VVDS overdensities $g\left(\delta_{\mathrm{g}}\right)$ reproduces the PDF of the underlying parent population of galaxies. The large size and high redshift sampling rate of the VVDS spectroscopic sample, together with the multi-color information in the $B, V, R, I$ filters of the parent photometric catalog and the relatively simple selection functions of the survey, allow us to check for the presence of observational systematics in the data. In principle, this analysis helps us to constrain the range of the parameter space where first-epoch VVDS data can be analyzed in a statistically unbiased way and results can be meaningfully interpreted.
The outline of the paper is the following: in Sect. 2 we briefly describe the first-epoch VVDS data sample. In Sect. 3 we introduce the technique applied for reconstructing the threedimensional density field traced by VVDS galaxies, providing details about corrections for various selection effects. In Sect. 4 we outline the construction of the PDF of galaxy overdensities and test its statistical representativity. We then derive the PDF of VVDS density contrasts and analyze its statistical moments. In Sect. 5 we review the theoretical properties of the analogous statistics for mass fluctuations. Particular emphasis is given to the problem of projecting the mass PDF derived in real space into redshift-perturbed comoving coordinates in the high redshift Universe. The method for computing the biasing function is introduced and tested against possible systematics in Sect. 6. VVDS results are presented and discussed in Sect. 7. and compared to theoretical models of biasing evolution in Sect. 8. Conclusions are drawn in Sect. 9.

The coherent cosmological picture emerging from independent observations and analysis motivate us to frame all the results presented in this paper in the context of a $\Lambda \mathrm{CDM}$ cosmological model with $\Omega_{\mathrm{m}}=0.3$ and $\Omega_{\Lambda}=0.7$. Throughout, the Hubble constant is parameterized via $h=H_{0} / 100$. All magnitudes in this paper are in the AB system (Oke \& Gunn 1983), and from now on we will drop the suffix $A B$.

\section{The first-epoch VVDS redshift sample}

The primary observational goal of the VIMOS-VLT Redshift Survey as well as the survey strategy and first-epoch observations in the VVDS-0226-04 field (from now on simply VVDS02h) are presented in Paper I.

In order to minimize selection biases, the VVDS survey in the VVDS-02h field has been conceived as a purely fluxlimited (17.5 $\leq I \leq 24)$ survey, i.e., no target pre-selection according to colors or compactness is implemented. Stars and QSOs have been a posteriori removed from the final redshift sample. Photometric data in this field are complete and free from surface brightness selection effects, up to the limiting magnitude $I=24$ (Mc Cracken et al. 2003).

First-epoch spectroscopic observations in the VVDS-02h field were carried out using the VIMOS multi-object spectrograph (Le Fèvre et al. 2003) during two runs between October and December 2002 (see Paper I). VIMOS observations have been performed using 1 arcsec wide slits and the LRRed grism which covers the spectral range $5500<\lambda(\AA)<9400$ with an effective spectral resolution $R \sim 227$ at $\lambda=7500 \AA$. The accuracy in redshift measurements is $\sim 275 \mathrm{~km} \mathrm{~s}^{-1}$. Details on observations and data reduction are given in Paper I, and in Le Fèvre et al. (2004).

The first-epoch VVDS-02h data sample extends over a sky area of $0.7 \times 0.7 \mathrm{deg}$ (which was targeted according to a 1,2 or 4 passes strategy, i.e., giving to any single galaxy in the field 1, 2 or 4 chances to be targeted by VIMOS masks (see Fig. 12 of Paper I) and has a median depth of about $z \sim 0.76$. It contains 6582 galaxies with secure redshifts (i.e., redshift determined with a quality flag $\geq 2$ (see Paper I)) and probes a comoving volume (up to $z=1.5$ ) of nearly $1.5 \times 10^{6} h^{-3} \mathrm{Mpc}^{3}$ in a standard 
$\Lambda \mathrm{CDM}$ cosmology. This volume has transversal dimensions $\sim 37 \times 37 h^{-1} \mathrm{Mpc}$ at $z=1.5$ and extends over $3060 h^{-1} \mathrm{Mpc}$ in radial direction.

For this study we define a sub-sample (VVDS-02h-4) with galaxies having redshift $z<1.5$ and selected in a continuous sky region of $0.4 \times 0.4 \mathrm{deg}$ which has been homogeneously targeted four times by VIMOS slitmasks. Even if we measure redshifts up to $z \sim 5$ and in a wider area, the conservative angular and redshift limits bracket the range where we can sample in a denser way the underlying galaxy distribution and, thus, minimize biases in the reconstruction of the density field (see the analysis in Sect. 4.1). The VVDS-02h-4 subsample contains 3448 galaxies with secure redshift (3001 with $0.4<z<1.5$ ) and probes one-third of the total VVDS-02h volume. This is the main sample used in this study.

\section{The density field reconstruction scheme}

The first ingredient we need in order to derive the biasing relation

$\delta_{\mathrm{g}}=b(z, \delta, R) \delta$

is a transformation scheme for diluting an intrinsic point-like process, such as the galaxy distribution in a redshift survey, into a continuous 3D overdensity field (see the review by Strauss \& Willick 1995). We write the dimensionless density contrast at the comoving position $\boldsymbol{r}$, smoothed over a typical dimension $R$ as

$\delta_{\mathrm{g}}(\boldsymbol{r}, R)=\frac{\rho_{\mathrm{g}}(\boldsymbol{r}, R)-\overline{\rho_{\mathrm{g}}}}{\overline{\rho_{\mathrm{g}}}}$.

and we define (e.g., Hudson 1993) the smoothed number density of galaxies above the absolute magnitude threshold $\mathcal{M}^{c}$ as the convolution between Dirac's delta functions and some arbitrary filter

$\rho_{\mathrm{g}}\left(\boldsymbol{r}, R,<\mathcal{M}^{c}\right)=\sum_{i} \frac{\delta^{D}\left(\boldsymbol{r}-\boldsymbol{r}_{i}\right) * F\left(\frac{\left|\boldsymbol{r}-\boldsymbol{r}_{i}\right|}{R}\right)}{S\left(r_{i}, \mathcal{M}^{c}\right) \Phi_{z}(m)}$

where the sum is taken over all the galaxies in the sample, $S\left(r, \mathcal{M}^{c}\right)$ is the distance-dependent selection (or incompleteness) function of the sample (see Sect. 3.2), $\Phi_{z}(m)$ is the redshift sampling function (see Sect. 3.3) and $F(|\boldsymbol{r}| / R)$ is a smoothing kernel of width $R$. In this paper, the smoothing window $F$ is modeled in terms of a normalized Top-Hat (TH) filter

$F\left(\frac{|\boldsymbol{r}|}{R}\right)=\frac{3}{\left(4 \pi R^{3}\right)} \Theta\left(1-\frac{|\boldsymbol{r}|}{R}\right)$,

where $\Theta$ is the Heaviside function, defined as $\Theta(x)=1$ for $0 \leq x \leq 1$, and $\Theta(x)=0$ elsewhere.

Within this weighting scheme, shot-noise errors are evaluated by computing the variance of the galaxy field

$\epsilon(\boldsymbol{r})=\frac{1}{\overline{\rho_{\mathrm{g}}}}\left[\sum_{i}\left(\frac{F\left(\frac{\left|r-r_{i}\right|}{R}\right)}{S\left(r_{i}, \mathcal{M}^{c}\right) \Phi_{z}\left(m_{i}\right)}\right)^{2}\right]^{1 / 2}$.

Note that all coordinates are comoving and that the mean density $\overline{\rho_{\mathrm{g}}}$ depends on cosmic time. Since we observe an evolution of nearly a factor of two in the mean density of galaxies brighter than $\mathcal{M}_{\mathrm{B}}^{*}(z=0)$ from redshift $z=0$ up to $z=1$ (see Ilbert et al. 2005, hereafter Paper II), we compute the value of $\overline{\rho_{\mathrm{g}}}$ at position $r$ (corresponding to some look-back time $t$ ) with Eq. (6) by simply averaging the galaxy distribution in survey slices $r \pm R_{\mathrm{S}}$ where $R_{\mathrm{S}}=400 h^{-1} \mathrm{Mpc}$. We note that conclusions drawn in this paper depend very weakly on the choice of $R_{\mathrm{S}}$ in the interval $200<R_{\mathrm{s}}\left(h^{-1} \mathrm{Mpc}\right)<600$.

In this paper, the density field is evaluated at positions $r$ in the VVDS-02h volume that can be either random or regularly displaced on a 3D grid (see Sect. 3.4). Even if we correct for the different sampling rate in the VVDS-02h field (by weighting each galaxy by the inverse of redshift sampling function $\left.\left(\Phi_{z}(m)\right)\right)$ we always select, for the purposes of our analysis, only the density fluctuations recovered in spheres having at least $70 \%$ of their volume in the denser 4-passes volume. This in order to minimize the Poissonian noise due to the sparser redshift sampling outside the VVDS-02h-4 field.

We also consider only volumes above the redshift threshold $z_{\mathrm{t}}$ where the transversal dimension $L$ of the first-epoch VVDS$02 \mathrm{~h}$ cone is $L\left(z_{\mathrm{t}}\right)>2 R$. As an example, for TH windows of size $R=5(10) h^{-1} \mathrm{Mpc}$ we have $z_{\mathrm{t}} \sim 0.4(0.7)$. Within the redshift range $0.4<z<1.5$ the VVDS-02h field contains 5252 galaxies (of which 3001 are in the four passes region).

Note that we have characterized the galaxy-fluctuation field in terms of the number density contrast, instead of the luminosity density contrast, because the former quantity is expected to show a time-dependent variation which is more sensitive to the galaxy evolution history (formation and merger rates, for example). Moreover, as described in Sect. 3.4, a robust description of the density field and a reliable determination of the PDF shape can be obtained only minimizing the shot noise component of the scatter; this is more easily done by considering galaxy number densities rather than galaxy light densities.

The most critical elements of the smoothing process are directly readable in Eq. (6): we must first evaluate galaxy absolute magnitudes at each redshift in the most reliable way, then specify the selection function and the redshift sampling rate of the VVDS survey. In the next sections we will describe how these quantities have been evaluated.

\subsection{The K-correction}

The absolute magnitude is defined as:

$M^{r}=m^{o}-5 \log d_{L}(z, \mathbf{\Omega})-K(z, \mathrm{SED})$

where the suffixes $r$ and $o$ designate respectively the rest-frame band in which the absolute magnitude is computed and the band where the apparent magnitude is observationally measured, and $d_{L}$ is the luminosity distance evaluated in a given a priori cosmology (i.e., using an appropriate set of cosmological parameters $\boldsymbol{\Omega} \equiv\left[\Omega_{\mathrm{m}}, \Omega_{\Lambda}\right]$ ).

The correction factor $K$, which depends on redshift and the spectral energy distribution (SED), accounts for the fact that the system response in the observed frame corresponds to a narrower, bluer rest-frame passband, depending on the redshift of the observed object. A complete description of the application of this transformation technique to VVDS galaxies is detailed in Paper II. 
The estimate of the galaxy absolute luminosity is thus affected by the uncertainties introduced by probing redshift regimes where the k-correction term cannot be neglected. Using mock catalogs simulating the VVDS survey, we have shown (see Fig. A.1 in Paper II) that the errors in the recovered absolute magnitude are significantly smaller in the $B$ band $\left(\sigma_{B}=0.08\right)$ than in the $I$ band $\left(\sigma_{I}=0.17\right)$. Thus, in what follows we will use absolute luminosities determined in the $B$-band rest-frame.

\subsection{The radial selection function}

Since our sample is limited at bright and faint apparent magnitudes $(17.5 \leq I \leq 24)$, at any given redshift we can only observe galaxies in a specific, redshift-dependent, absolute magnitude range. It is usual to describe the sample radial incompleteness by defining the selection function in terms of the galaxy luminosity function $\varphi(\mathcal{M})$

$S\left(r, \mathcal{M}^{c}\right)=\frac{\int_{\mathcal{M}_{b}(r)}^{\mathcal{M}_{f}(r)} \varphi(\mathcal{M}) \mathrm{d} \mathcal{M}}{\int_{-\infty}^{\mathcal{M}^{c}} \varphi(\mathcal{M}) \mathrm{d} \mathcal{M}}$.

Here $\mathcal{M}_{b}(r)$ and $\mathcal{M}_{f}(r)$ are the $B$-band absolute magnitudes which correspond, at distance $r$, to the $I$-band limiting apparent magnitudes $m_{b}=17.5$ and $m_{f}=24$ respectively (see discussion at the end of the section). Since Eq. (9) also depends on galaxy colors, we compute its mean value at distance $r$ by a weighted average over the population mix observed at distance $r$.

The VVDS luminosity function (LF) has been derived in Paper II and is characterized by a substantial degree of evolution over the redshift range $0<z<1.5$. Therefore, we estimate $\varphi(\mathcal{M})$ in the $B$ band at any given position in the redshift interval $[0,1.5]$ by interpolating, with a low order polynomial function, the Schechter shape parameters $\alpha$ and $\mathcal{M}^{*}$ given in Table 1 of Paper II.

Assuming $\mathcal{M}_{B}^{c}=-15$ in Eq. (10), which corresponds to the limiting absolute magnitude over which the LF of the VVDS$02 \mathrm{~h}$ sample can be robustly constrained in the lowest redshift bins, the selection function exponentially falls by nearly 2 orders of magnitude in the redshift range up to $z<1.5$. Thus, the density field reconstruction strongly depends on the radial selection function used especially at high redshifts, where Eq. (10) can be affected by possible systematics in the determination of the LF or in the measurements of faint magnitudes. Therefore, we will also analyze volume-limited sub-samples, which are essentially free from these systematics.

Since in a magnitude limited survey progressively brighter galaxies are selected as a function of redshift, a volume-limited sample also allows us to disentangle spurious luminositydependent effects from the measurement of the redshift evolution of the biasing function.

\subsection{The redshift sampling rate}

As for most redshift surveys, the VVDS does not target spectroscopically all the galaxies that satisfy the given flux limit criteria in the selected field of view (see Paper I). Because of

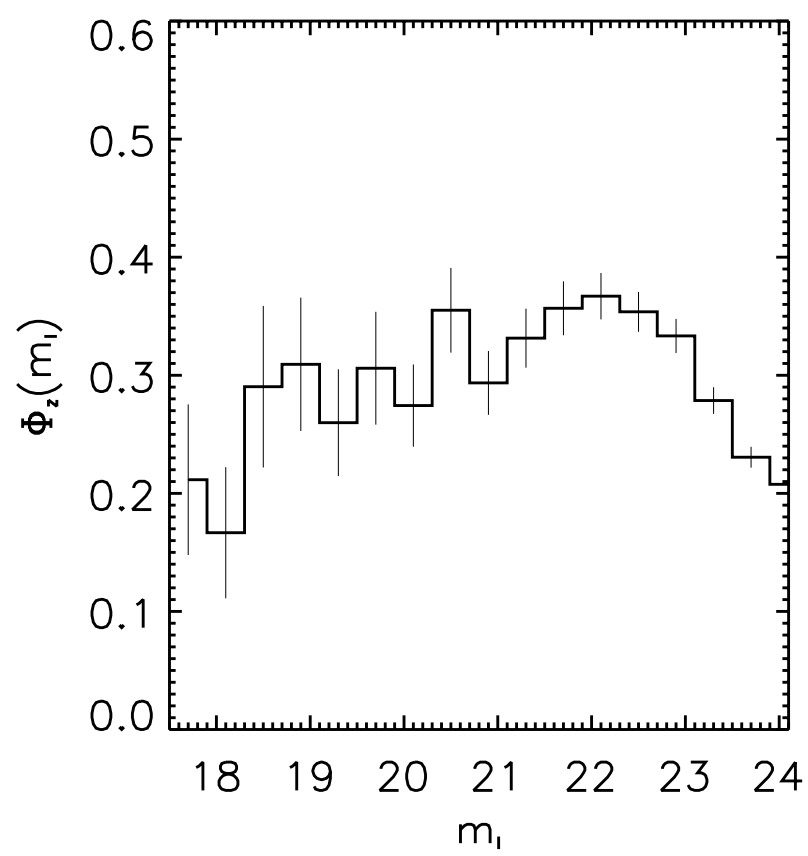

Fig. 1. The VVDS redshift sampling rate in the four-passes VVDS$02 \mathrm{~h}-4$ region is plotted versus the observed apparent magnitude in the $I$-band. The mean redshift sampling rate is $\sim 0.3$.

the sparse sampling strategy, we have to correct the density estimator with a sampling rate weight $\Phi_{z}$ in order to reconstruct the real underlying galaxy density field in a statistically unbiased way.

The VVDS redshift sampling rate is the combination of two effects: i) only a fraction of the galaxies ( 40\%, see Paper I) in the photometric sample is targeted (target sampling rate); ii) and only a fraction of the targeted objects ( $\sim 80 \%$ see Paper I) yield a redshift (spectroscopic success rate). We can model this correction term, by assuming, to a first approximation, that the sampling rate depends only on the apparent magnitude. Since the VVDS targeting strategy is optimized to maximize the number of slits on the sky, the selection of faint objects is systematically favored. Inversely, the ability of measuring a redshift degrades progressively towards fainter magnitudes, i.e., for spectra having lower signal-to-noise ratios (the spectroscopic success rate decreases from $>90 \%$ at $I \sim 22$ down to $\sim 60 \%$ at $I \sim 24)$. These two opposite effects conspire to produce the magnitude-dependent sampling rate function shown in Fig. 1. Clearly, with such an approximation, we neglect any possible dependence of the sampling rate from other important parameters such as for example surface brightness, spectral type or redshift. However, in Sect. 3.2 of Paper II we showed, using photometric redshifts, that any systematic sampling bias introduced by a possible redshift dependence of the spectroscopic success rate is expected to affect only the tails of our observed redshift distribution $(z<0.5$ and $z>1.5)$ i.e., redshift intervals not considered in this study (see Sect. 3).

We describe the VVDS sampling completeness $\Phi_{z}(m)$ at a given magnitude $m$, as the fraction of objects with measured 
redshifts $N_{z}$ over the number $N$ of objects detected in the photometric catalog.

$\Phi_{z}(m)=\frac{\sum_{i=1}^{N_{z}} w\left(m-m_{i}, d m\right)}{\sum_{i=1}^{N} w\left(m-m_{i}, d m\right)}$

with the window function $w$ defined as:

$w\left(m-m_{i}, d m\right)= \begin{cases}1 & \text { if }\left|m-m_{i}\right|<d m / 2 \\ 0 & \text { otherwise }\end{cases}$

Working in low resolution mode (i.e. allowing up to 4 galaxies to have spectra aligned along the same dispersion direction with a typical sky separation of 2 arcmin) and observing the same region of sky 4 times for a total of about 16 hours (fourpasses strategy), we can measure redshifts for nearly $30 \%$ of the parent photometric population, as shown in Fig. 1. In other terms, on average, nearly one over three galaxies with magnitude $I \leq 24$ has a measured redshift in the four-passes VVDS region. This high spatial sampling rate is a critical factor for minimizing biases in the reconstruction of the 3D density field of galaxies. In particular we note that our $I \leq 24, z<1.5$ VVDS-02h-4 sample is characterized by an effective mean inter-particle separation in the redshift range $[0,1.5](\langle r\rangle \sim$ $\left.5.1 \mathrm{~h}^{-1} \mathrm{Mpc}\right)$ which is smaller than that of the original CFA sample $\left(\langle r\rangle \sim 5.5 h^{-1} \mathrm{Mpc}\right)$ used by Davis \& Huchra (1981) to reconstruct the $3 \mathrm{D}$ density field of the local Universe (i.e., out to $\sim 80 h^{-1} \mathrm{Mpc}$ ). At the median depth of the survey, i.e., in the redshift interval $0.75 \pm 0.05$, the mean inter-particle separation is $4.4 h^{-1} \mathrm{Mpc}$, a value nearly equal to the mean inter-particle separation at the median depth of the $2 \mathrm{dFGRS}$. We finally note that in the redshift range $[0.7,1.35]$, which is also covered by the DEEP2 survey, the VVDS mean-inter-particle separation is $5.5 h^{-1} \mathrm{Mpc}$ compared to the value $\sim 6.5 h^{-1} \mathrm{Mpc}$ inferred from the values quoted by Coil et al. (2004) for their most complete field, currently covering $0.32 \mathrm{deg}^{2}$.

By dividing the VVDS-02h-4 field in smaller cells and repeating the analysis, we conclude that the sampling rate does not show appreciable variations, i.e., the angular selection function can be considered constant for the purposes of our analysis. This corresponds to the fact that the success rate in redshift measurement in each VIMOS quadrant (i.e., the spectroscopic success rate per mask) is, to a good approximation, constant and equal to $\sim 80 \%$ (see Paper I).

\subsection{Shot noise}

In a flux-limited sample, the shot noise in the density field is an increasing function of distance (see Eq. (8)). One may correct for the increase of the mean VVDS inter-particle separation as a function of redshift (and thus the increase of the variance of the density field) by opportunely increasing the length of the smoothing window (e.g., Strauss \& Willick 1995). However, since we are interested in comparing the fluctuations recovered on the same scale at different redshifts in a flux limited survey, we take into account the decreased sampling sensitivity of the survey at high redshift in an alternative way.

We deconvolve the signature of this noise from the density maps by applying the Wiener filter technique

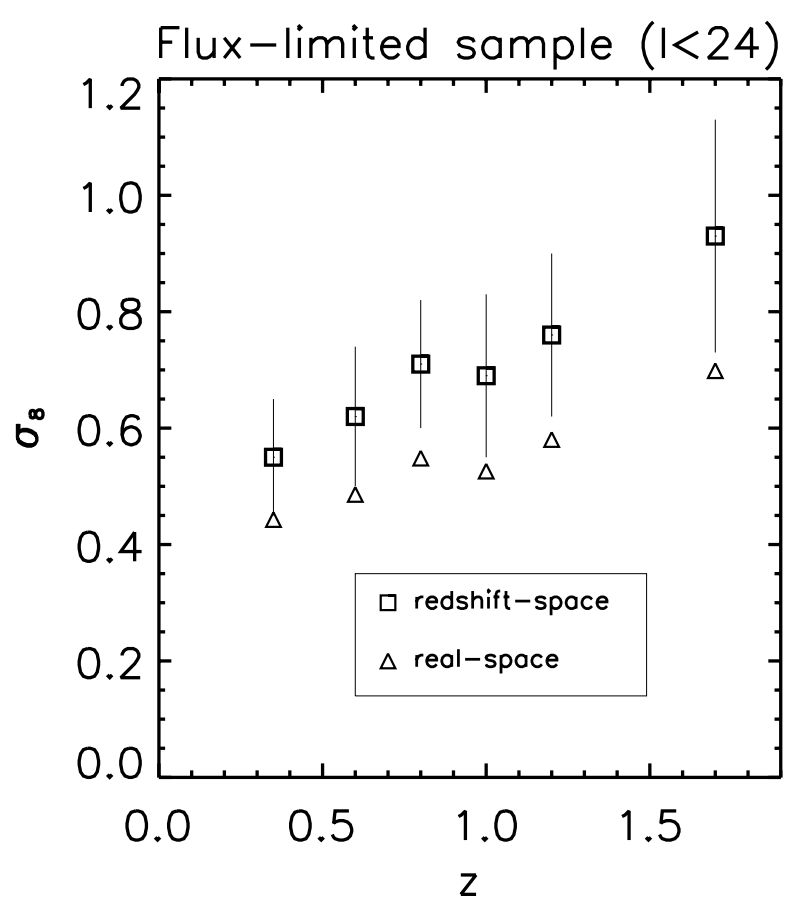

Fig. 2. The real- and redshift-space rms fluctuations of the flux-limited VVDS sample recovered using Eq. (13) and the results of the correlation function analysis presented in Paper III are plotted at six different redshifts in the interval $0.4<z<1.7$.

(cf. Press et al. 1992) which provides the minimum variance reconstruction of the smoothed density field, given the map of the noise and the a priori knowledge of the underlying power spectrum (e.g., Lahav et al. 1994). The application of the Wiener denoising procedure to the specific geometry of the VVDS sample is described in detail in Appendix A.

Here we note that the Wiener filter requires a model for the underlying $3 \mathrm{D}$ power spectrum $P(k, z)$ which we compute, over the frequency scales where the correlation function of VVDS galaxies is well constrained $(0.06 \leq k \leq 10)$, as (see Eq. (48) in Appendix A):

$P(k, z)=4 \pi \frac{r_{0}(z)^{\gamma(z)}}{k^{3-\gamma(z)}} \Gamma(2-\gamma(z)) \sin \frac{\gamma(z) \pi}{2}$,

where the normalization $r_{0}(z)$ and the slope $\gamma(z)$ of the correlation function at redshift $z$ have been derived by interpolating the values measured in various redshift intervals of the VVDS$02 \mathrm{~h}$ volume by le Fèvre et al. (2005, hereafter Paper III).

The variance of the galaxy distribution on a $8 h^{-1} \mathrm{Mpc}$ scale in the VVDS-02h sample can be obtained by integrating Eq. (13) using a TH window of radius $8 h^{-1} \mathrm{Mpc}$ and the $\left(r_{0}, \gamma\right)$ parameters of the VVDS correlation function

$\sigma_{8}^{2}=\frac{1}{2 \pi^{2}} \int P(k) \tilde{F}_{k}^{2} k^{2} \mathrm{~d} k$

where $\tilde{F}_{k}$ is the Fourier transform of the TH filter (see Eq. (45) in Appendix A). Note that, by integrating the power spectrum down to $k \rightarrow 0$, i.e., extrapolating the power law shape of Eq. (13) beyond $L \sim 100 h^{-1} \mathrm{Mpc}(k \lesssim 0.06)$, one would revise upwards the value of $\sigma_{8}$ by $\sim 2 \%$ (at $z=0.35$ ) and by $\sim 4 \%$ at $z=1.4$. Since, however, the amplitude of the power spectrum 


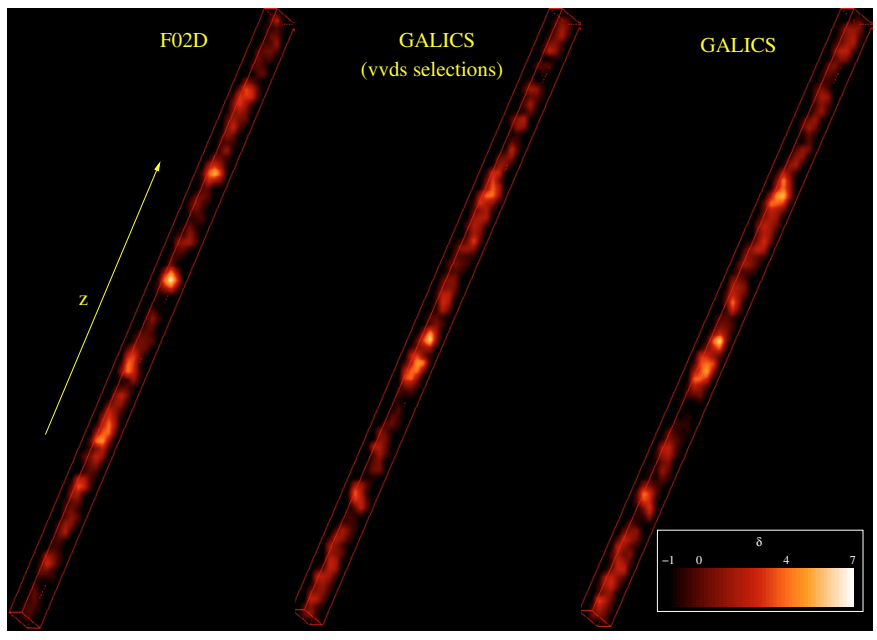

Fig. 3. 3D density field traced by the galaxy distribution in the VVDS02h Field (left, 1641 galaxies), in the flux-limited $(I \leq 24)$ GALICS simulation (right, 9450 galaxies), and in the flux-limited GALICS sample after applying the VVDS target selection criteria (center, 1656 galaxies). Data span the redshift interval $[0.8,1.1]$. In each cone, the galaxy distribution is continuously smoothed using a TH window function with $R=5 h^{-1} \mathrm{Mpc}$ which nearly corresponds to the mean inter-particle separation in this same redshift interval. The cone metric was computed assuming a $\Lambda \mathrm{CDM}$ cosmology and the correct axis ratio between transversal and radial dimensions has been preserved. The cones have approximate transverse dimensions of $28 h^{-1} \mathrm{Mpc}$ at $z=1$ and extend over $527 \mathrm{~h}^{-1} \mathrm{Mpc}$.

on large scales is expected to downturn and to be systematically lower than predicted by Eq. (13), we safely conclude that, with our computation scheme, the inferred $\sigma_{8}$ value should be biased low by no more than $\sim 2 \%$ and $4 \%$ in the first and last redshift bin, respectively.

Projecting the results from real-space into the redshiftdistorted space (see Sect. 5), i.e., implementing the effects of large-scale streaming motions, we infer that the rms of galaxy fluctuations are $\sigma_{8}=[0.55 \pm 0.10,0.62 \pm 0.12$, $0.71 \pm 0.11,0.69 \pm 0.14,0.75 \pm 0.14,0.92 \pm 0.20]$ at redshift $z=[0.35,0.6,0.8,1.2,1.2,1.65]$ (see Fig. 2). Thus, the amplitude of $\sigma_{8}$ for a flux-limited $I \leq 24$ sample increases as a function of redshift by nearly $70 \%$ between $z \sim 0.3$ and $z \sim 1.7$.

\subsection{The 3-dimensional VVDS density field}

The VVDS-02h galaxy density field reconstructed on a scale $R=5 h^{-1} \mathrm{Mpc}$ and in the redshift bin $0.8<z<1.1$ is visually displayed in the left most panel of Fig. 3. Note that the chosen smoothing scale nearly corresponds to the mean inter-galaxy separation in the VVDS-02h sample at $z \sim 1$. Figure 3 shows the regular patterns traced by over- and under-dense regions in the selected redshift interval. Specifically, we note that, in this redshift slice, there are over- and under-dense regions which extend over characteristic scales as large as $\sim 100 h^{-1} \mathrm{Mpc}$. A more complete discussion of the "cartography" in such deep regions of the Universe is presented by Le Fèvre et al. (2005c).

In the same figure, we also display the density field reconstructed in an analogous redshift range, using the GALICS simulation (Galaxies in Cosmological Simulations, Hatton et al. 2003). GALICS combines cosmological simulations of dark matter with semi-analytic prescriptions for galaxy formation to produce a fully realistic deep galaxy sample. In particular we plot the density field of the $I \leq 24$ flux-limited simulation as well as the density field recovered after applying to the pure flux-limited simulation all the VVDS target selection criteria (see Sect. 4.1). No qualitative difference between the density fields reconstructed before and after applying to the simulation all the survey systematics is seen.

Clearly, a more quantitative assessment of the robustness and reliability of the VVDS overdensity field can be done by studying its PDF.

\section{The PDF of galaxy fluctuations}

Once the three-dimensional field of galaxy density contrasts $\delta_{\mathrm{g}}$ has been reconstructed on a given scale $R$, one can fully describe its properties by deriving the associated PDF $g\left(\delta_{\mathrm{g}}\right)$. This statistical quantity represents the normalized probability of having a density fluctuation in the range $\left(\delta_{\mathrm{g}}, \delta_{\mathrm{g}}+\mathrm{d} \delta_{\mathrm{g}}\right)$ within a region of characteristic length $R$ randomly located in the survey volume.

While the shape of the PDF of mass fluctuations at any given cosmic epoch is theoretically well constrained from CDM simulations (see next section), little is known about the observational PDF of the general population of galaxies in the high redshift Universe. Even locally, this fundamental statistics has been often overlooked (but see Ostriker et al. 2003). Notwithstanding, the shape of the galaxy PDF is strongly sensitive to the effects of gravitational instability and galaxy biasing, and its redshift dependence encodes valuable information about the origin and evolution of galaxy density fluctuations.

The shape of the PDF can be characterized in terms of its statistical moments. In particular the variance of a zero-mean field (such as the overdensity field we are considering) is

$\sigma_{R}^{2}=\left\langle\delta_{\mathrm{g}}^{2}\right\rangle_{R} \equiv \int_{0}^{\infty} \delta_{\mathrm{g}}^{2} g_{R}\left(\delta_{\mathrm{g}}\right) \mathrm{d} \delta_{\mathrm{g}}$

Higher moments can be straightforwardly derived (see Bernardeau et al. 2002, for a review). In the following, we will drop the suffix $R$, unless we need to emphasize it.

\subsection{Estimating reconstruction systematics using mock catalogs}

For the purposes of our analysis, it is imperative to check that the various instrumental selection effects as well as the VVDS observing strategy are not compromising the determination of the PDF of galaxy fluctuations. In this section, we explore the region of the parameter space (essentially redshift and smoothing scales) where the PDF of VVDS overdensities traces in a statistically unbiased way the underlying parent distribution.

Possible systematics can be hidden in the reconstructed PDF essentially because i) the VVDS redshift sampling rate is not unity; ii) the slitlets are allocated on the VIMOS masks with different constraints along the dispersion and the spatial 
axis, and iii) the VIMOS field of view is splitted in four different rectangular quadrants separated by a vignetting cross.

We have addressed point iii) by designing a specific telescope pointing strategy which allows us to cover in a uniform way the survey sky region (see the telescope pointing strategy shown in Fig. 1 of Paper I). With the adopted survey strategy, we give to each galaxy in the VVDS-02h-4 field four chances to be targeted by VIMOS, thus increasing the survey sampling rate (nearly 1 galaxy over 3 with magnitude $I \leq 24$ has a measured redshift).

Concerns about points i) and ii) can be directly addressed using galaxy simulations covering a cosmological volume comparable to the VVDS one. Thanks to the implementation of the Mock Map Facility (MoMaF, Blaizot et al. 2003), it is possible to convert the GALICS 3D mocks catalog into $2 \mathrm{D}$ sky images, and handle the $2 \mathrm{D}$ projection of the simulation as a pseudo-real imaging survey. Pollo et al. (2005), have then built a set of 50 fully realistic mock VVDs surveys from the GALICS simulations to which the whole observational pipeline and biases has been applied. By comparing specific properties of the resulting pseudo-VVDS sample with the true underlying properties of the pseudo-real Universe from which the sample is extracted, we can directly explore the robustness, as well as the limits, of the particular statistical quantities we are interested in.

In brief these include addeding to the 3D galaxy mocks a randomly simulated distribution of stars to mimic the same star contamination affecting our survey. Next, we have masked the sky mocks using the VVDS photometric masks, i.e., we have implemented the same geometrical pattern of excluded regions with which we avoid to survey sky regions contaminated by the presence of bright stars or photometric defects. Then, we have extracted the spectroscopic targets by applying the target selection code (VMMPS, Bottini et al. 2005) to the simulated 2D sky distribution. To each GALICS redshift, which incorporates the Doppler contribution due to galaxy peculiar velocities, we have added a random component to take into account errors in $z$ measurements. Finally, we have processed the selected objects implementing the same magnitude-distribution of failures in redshift measurements which characterizes the first-epoch observations of the VVDS survey (see Paper I and Fig. 1).

Since GALICS galaxies have magnitudes simulated in the same 4 bands surveyed by $\operatorname{VVDS}(B, V, R, I)$, we have applied the K-correction to obtain rest frame absolute magnitudes and we have empirically re-derived all the selection functions for the mock catalogs according to the scheme presented in Sect. 3. In this way we can also check the robustness of the techniques we apply for computing absolute magnitudes and selection functions (see Paper II).

The PDF of galaxy overdensities obtained from the mock samples has been finally compared to the PDF of the parent population. For brevity, in what follows, we will call $s$-samples (survey-samples) the mocks simulating the VVDS redshift survey and $p$-sample (parent sample) the whole GALICS simulation flux-limited at $17.5 \leq I \leq 24$.

The density contrasts have been calculated as described in Sect. 3. In the following, we will restrict our analysis to the set of smoothing scales in the interval $R=(5,10) h^{-1} \mathrm{Mpc}$. The choice of these particular limits is motivated by the fact that $5 h^{-1} \mathrm{Mpc}$ is the minimum smoothing scale for which the reconstructed density field is unbiased over a substantial redshift interval (see discussion below). Note, also, that below this typical scale, linear regimes approximations, largely used in this paper, do not hold anymore. The upper boundary is constrained by the transverse comoving dimensions covered by the firstepoch VVDS data (see Sect. 2), which is still too small for being partitioned using bigger scale-lengths without introducing significant noise in the reconstructed PDF (see the transverse comoving dimension of the VVDS-02h field quoted in Sect. 2).

The PDF of the galaxy density contrasts computed using the s-sample is compared to the parent distribution inferred from the p-sample in Fig. 4. We conclude that the distribution of galaxy overdensities of the s-samples for $R=8$ and $10 h^{-1} \mathrm{Mpc}$ scales is not biased with respect to the underlying distribution of p-sample galaxy fluctuations. Thus, the VVDS density field reconstructed on these scales is not affected by the specific VVDS observational strategy.

It is evident in Fig. 4 that the VVDS redshift sampling rate is not high enough to map in an unbiased way the low density regions of the Universe $\left(\log \left(1+\delta_{\mathrm{g}}\right) \lesssim-0.5\right)$ when the galaxy distribution is smoothed on scales of $5 h^{-1} \mathrm{Mpc}$. Figure 5 shows that incompleteness in underdense regions is a function of redshift, with the bias in the low-density tail of the PDF developing and increasing as the redshift increases. As a rule of thumb, the PDF of the s-sample starts to deviate significantly from the parent PDF when the mean inter-galactic separation $\langle r\rangle$ of the survey sample is larger than the scale $\mathrm{R}$ on which the field is reconstructed. Since we measure $\langle r(z=1)\rangle \sim 5 h^{-1} \mathrm{Mpc}$ $\left(\langle r(z=1.5)\rangle \sim 8 h^{-1} \mathrm{Mpc}\right)$, the PDF of the density field recovered using a $\mathrm{TH}$ filter of radius $5 h^{-1} \mathrm{Mpc}$ is effectively unbiased (at least over the density range we are interested in, i.e., $\log (1+\delta)>-1)$ only if the sample is limited at $z \lesssim 1$. Therefore, in the following, results obtained for $R=5 h^{-1} \mathrm{Mpc}$ are quoted only up to $z=1$.

On scales $R>8 h^{-1} \mathrm{Mpc}$, the agreement between the PDFs of s- and p-samples holds true also for volume-limited subsamples Specifically, the 2nd and 3rd moments of the PDF of overdensities recovered using volume-limited $s$-samples on these scales are within $1 \sigma$ of the corresponding values computed for the parent, volume-limited, $p$-samples in each redshift interval of interest up to $z=1.5$.

To summarize, the results of simulated VVDS observations presented in this section show that, at least on scales $R \geq 8 h^{-1} \mathrm{Mpc}$, the VVDS PDF describes in an unbiased way the general distribution properties of a sample of $I=24$ flux-limited galaxies up to redshift 1.5. In other terms, the VVDS density field sampled in this way is essentially free from selection systematics in both low- and high-density regions, and can be meaningfully used to infer the physical bias in the distribution between galaxy and matter. Obviously, the representativeness of the measured PDF of VVDS overdensities with respect to the "universal" PDF up to $z=1.5$ is a different question. Since the volume probed is still restricted to a limited region of space in one field, the shape and moments of the galaxy PDF derived from the VVDS-02h are expected 


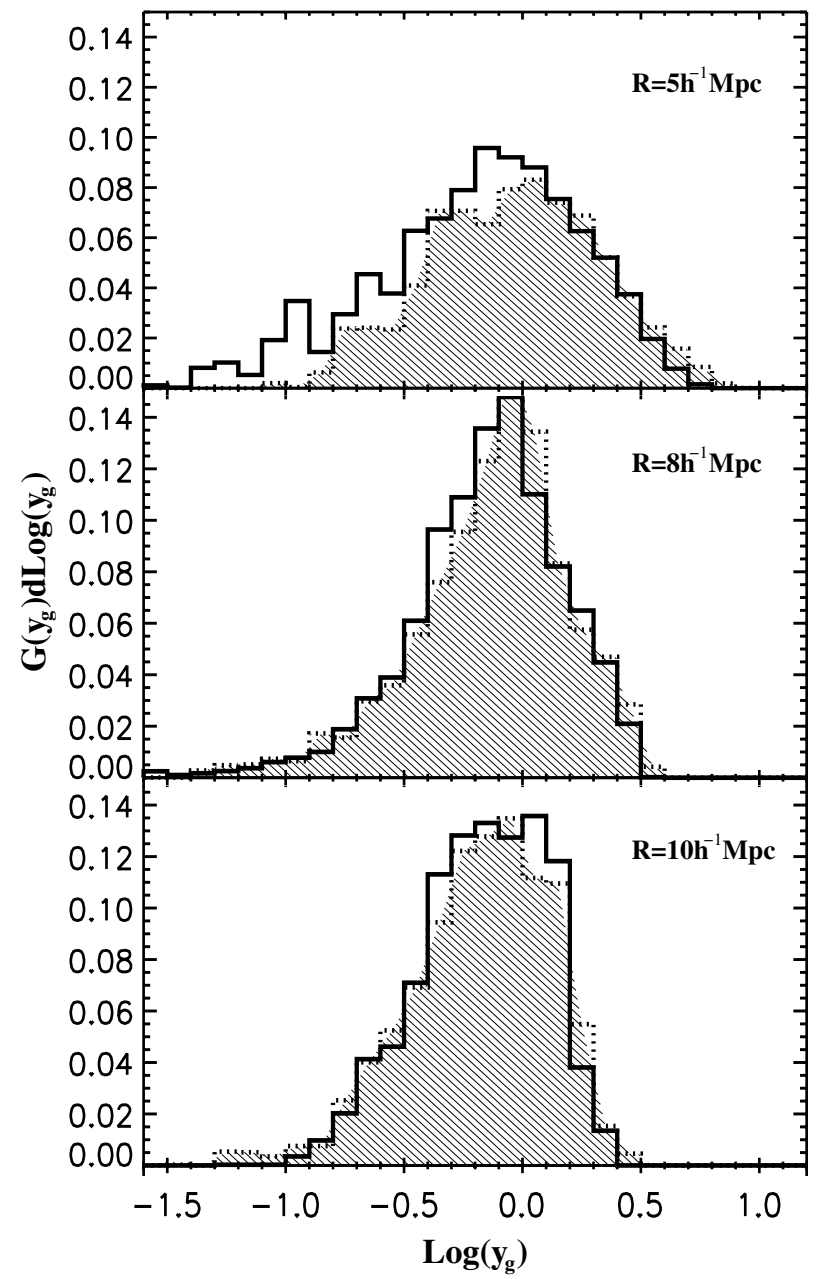

Fig. 4. Tests of the PDF reconstruction scheme using the mocks VVDS samples extracted from GALICS. The differential $\left(G\left(y_{\mathrm{g}}\right)\right)$ probability distribution functions of $y_{\mathrm{g}}=1+\delta_{\mathrm{g}}$ for the "observed" s-sample (dotted line, shadowed histogram), and for the parent p-sample (solidline). Note that the plotted histograms actually corresponds to $G\left(y_{\mathrm{g}}\right)=$ $\left.\ln (10) y_{\mathrm{g}} g\left(y_{\mathrm{g}}\right)\right)$ since the binning is done in $\log \left(y_{\mathrm{g}}\right)$. The logarithmic PDFs are computed for density fields smoothed using TH filters of different sizes (indicated on the top of each panel).

to deviate from the "universal" PDF of galaxies at this redshift because of cosmic variance. Reducing the cosmic variance is one of the main goals of extending the VVDS to 4 independent fields. Anyway, our 50 mock realisations of the VVDS$02 \mathrm{~h}$ sample allow us to estimate realistic errors that include the contribution from cosmic variance.

\subsection{The PDF of VVDS galaxies: results}

Let us then investigate the evolution, as a function of the lookback time, of the observed PDF of VVDS galaxy fluctuations.

In an apparent magnitude-limited survey such as the VVDS, only brighter galaxies populate the most distant redshift bins, whereas fainter galaxies are visible only at lower redshifts. As more luminous galaxies tend to cluster more strongly than fainter ones (e.g., Hamilton 1988; Croton et al. 2004), the

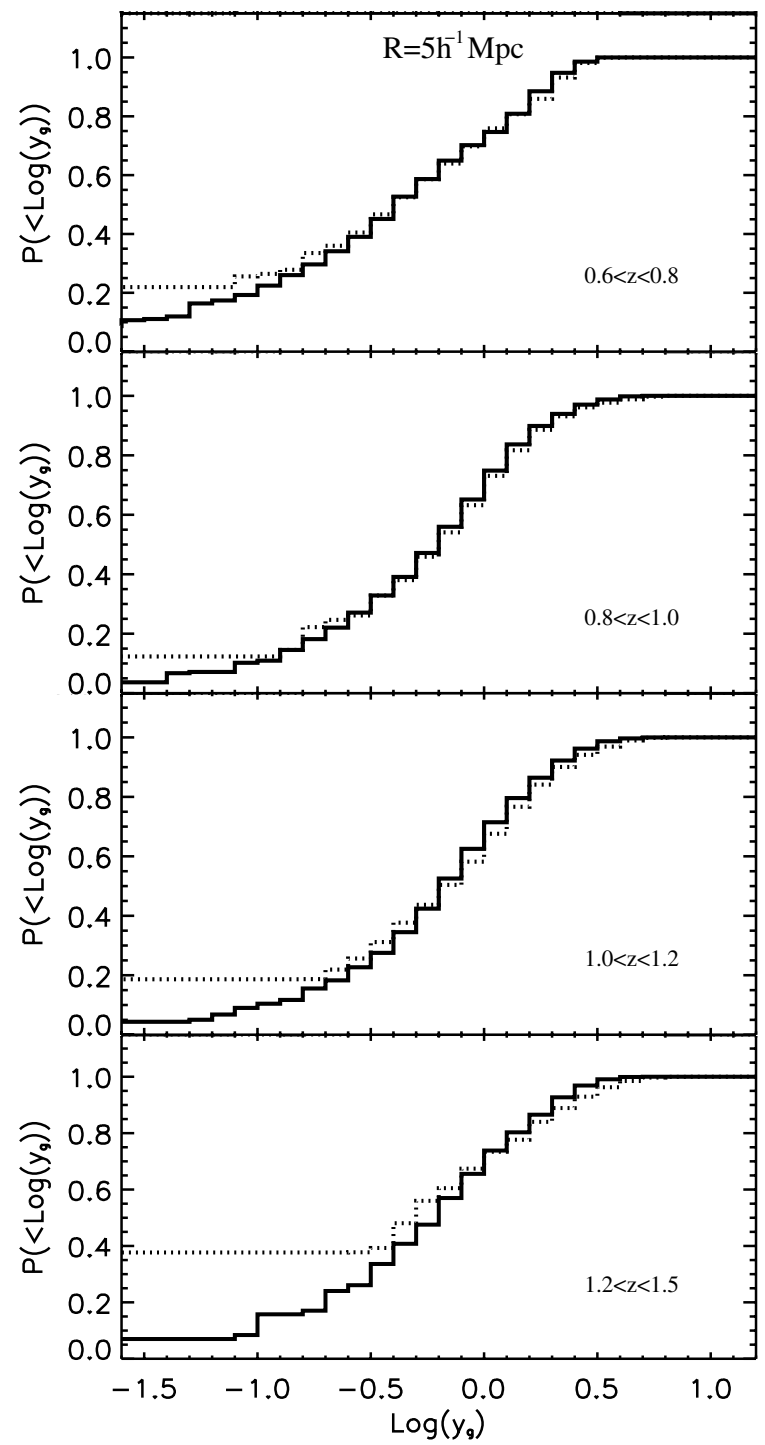

Fig. 5. The cumulative distribution function of density contrasts $y_{\mathrm{g}}=$ $1+\delta_{\mathrm{g}}$, on scales $R=5 \mathrm{~h}^{-1} \mathrm{Mpc}$, as recovered in different redshift intervals for the s-sample (dotted line), and for the parent p-sample (solid-line). The flattened pedestal at the low-density end of the cumulative distribution is due to low density regions in the p-sample that are spuriously sampled as empty regions $\left(\delta_{\mathrm{g}}=-1\right)$ in the s-sample.

PDF of galaxy fluctuations is expected to be systematically biased as a function of redshift.

This effect is clearly seen in the first correlation analysis of the VVDS (Paper III), and can be minimized by selecting a volume-limited sample. Therefore, we have defined a subsample with absolute magnitude brighter than $\mathcal{M}_{B}^{c}=$ $-20+5 \log h$ in the rest frame $B$ band $(\sim 1350$ galaxies with $0.7<z<1.5$ in the VVDS-02h field, $\sim 800$ of which are in the VVDS-02h-4 region).

This threshold corresponds to the faintest galaxy luminosity visible at redshift $z=1.5$ in a $I=24$ flux-limited survey and it is roughly 0.6 magnitudes brighter(fainter) than the value of $\mathcal{M}_{B}^{*}$ recovered at $z \sim 0(\sim 1.5)$ using the VVDS data (see Paper II). The median absolute magnitude for this volumelimited sample is $\sim-20.4$. 

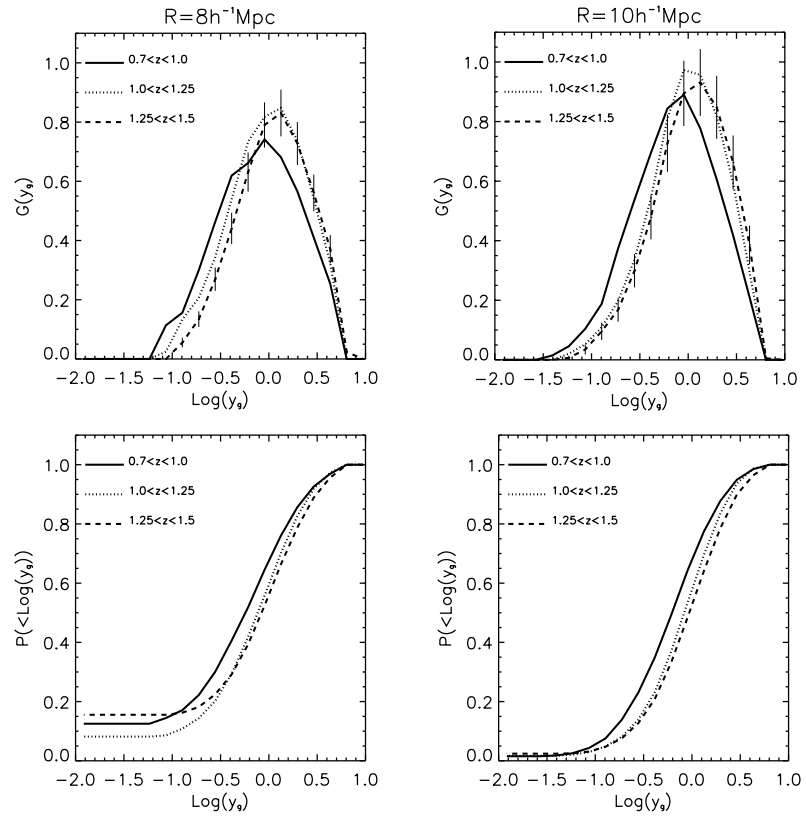

Fig. 6. Top: the PDF per units of galaxy overdensities $(G(y)=$ $\ln (10) y g(y))$ is plotted for the volume limited VVDS sample $\left(\mathcal{M}_{B}^{c}=\right.$ $-20+5 \log h)$ at three different redshifts. The PDFs are computed for density fields recovered using TH filters of size 8 and $10 \mathrm{~h}^{-1} \mathrm{Mpc}$. Bottom: the corresponding cumulative distributions. Errorbars (which, for clarity, are plotted only for the redshift bin $1.25<z<1.5$ ) represent the Poissonian uncertainties. The flattened pedestal at the lowdensity end of the cumulative distribution is contributed by empty regions $\left(\delta_{\mathrm{g}}=-1\right)$.

We note, however, that the populations of galaxies with the same luminosity at different redshifts may actually be different. As we have shown in Paper II, we measure a substantial degree of evolution in the luminosity of galaxies, and, as a consequence, with our absolute magnitude cut we are selecting $\mathcal{M}^{*}+0.6$ galaxies at $z=1.5$, but $\mathcal{M}^{*}-0.6$ galaxies at $z=0$. Thus, the clustering signal at progressively earlier epochs may not be contributed by the progenitors of the galaxies that are sampled at later times in the same luminosity interval.

The PDF of density fluctuations, in various redshift intervals, and traced on scales of 8 and $10 h^{-1}$ Mpc by VVDS galaxies brighter than $\mathcal{M}_{B}^{c}$, is presented in Fig. 6. Note that the analysis of the previous section guarantees that, on these scales, the VVDS PDF fairly represents the PDF of the real underlying population of galaxies up to $z=1.5$. Figure 6 shows how the shape of the measured galaxy PDF changes across different cosmic epochs.

A Kolmogorov-Smirnov test confirms that the PDFs at different cosmic epochs are statistically different (i.e., the null hypothesis that the three distributions are drawn from the same parent distribution is rejected with a confidence $P_{\mathrm{ks}}>1-10^{-6}$ ).

In particular the peak of the PDF in the lowest redshift interval is shifted towards smaller values of the density contrast $\delta_{\mathrm{g}}$ when compared to the peak of the PDF in the highest redshift bin. Moreover, the shape of the distribution, also shows a systematic "deformation". Specifically, we observe the development of a low- $\delta$ tail in the PDF as a function of time on both scales investigated. In other terms the probability of having low

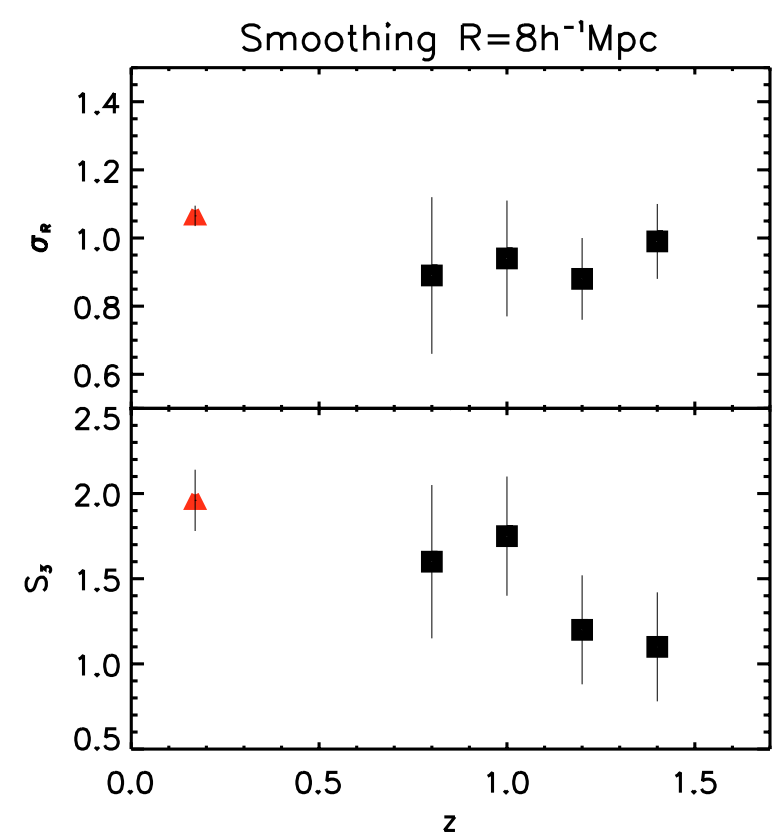

Fig. 7. Redshift evolution of the standard deviation (upper panel) and of the skewness (lower panel) of the galaxy PDF on a scale $R=8 h^{-1} \mathrm{Mpc}$ for galaxies brighter than $\mathcal{M}=-20+5 \log h$. The corresponding local values, estimated on the same scale by Croton et al. (2004) using a subsample of the 2dFGRS having nearly the same median absolute luminosity of our sample, are represented with triangles. Error bars represent $1 \sigma$ errors, and, in the case of VVDS measurements, include the contribution from cosmic variance. The errorbar on $\sigma_{8}$ of the $2 \mathrm{dFGRS}$ is smaller than the symbol size.

density regions increases as a function of cosmic time. For example, underdense regions, defined as the regions where the galaxy density field is $\log \left(1+\delta_{\mathrm{g}}\right)<-0.5$ on a $R=8 h^{-1} \mathrm{Mpc}$ scale, occupy a fraction of nearly $35 \%$ of the VVDS volume at redshift $0.7<z<1.0$, but only a fraction of about $25 \%$ at redshift $1.25<z<1.5$. Similar trends are observed when lowering the absolute luminosity threshold of the volume limited sample (and consequently lowering the upper limit of the redshift interval probed) or when modifying the binning in redshift space.

If galaxies are faithful and unbiased tracers of the underlying dark matter field, then both this effects, the peak shift and the development of a low density tail may be qualitatively interpreted as a direct supporting evidence for the paradigm of the evolution of gravitational clustering in an expanding Universe. At variance with overdense regions which collapse, a net density deficit $(\delta<0)$ in an expanding Universe brings about a sign reversal of the effective gravitational force: a density depression is a region that induces an effective repulsive peculiar gravity (Peebles 1980). If gravity is the engine which drives clustering in an expanding Universe, we thus expect that, as time goes by, low density regions propagate outwards and a progressively higher portion of the cosmological volume becomes underdense.

The observed evolution in the PDF could also indicate the presence of a time-dependent biasing between matter and galaxies. As a matter of fact, it can be easily shown that a monotonic bias, increasing with redshift, offers a natural mechanism 
to re-map the galaxy PDF into progressively higher intervals of density contrasts.

We can better discriminate the physical origin of the observed trends, i.e., if they are purely induced by gravitation or strengthened by the collateral and cooperative action of biasing, by studying the evolution of the PDF moments. In Fig. 7 the redshift evolution of the rms $(\sigma)$ and skewness $\left(S_{3}\right)$ of the overdensity fields (see Table 1 ) for the $\mathcal{M}_{B}<-20+5 \log h$ sample are shown and compared to local measurements.

Following the standard convention within the hierarchical clustering model, we define the skewness $S_{3}$ in terms of the volume-averaged two- and three-point correlation functions $\left(S_{3} \equiv \bar{\xi}_{3} / \bar{\xi}_{2}^{2}\right)$ noting that in the case of a continuous $\delta_{\mathrm{g}}$-field with zero mean this expression reduces to $S_{3} \equiv\left\langle\delta_{\mathrm{g}}^{3}\right\rangle /\left\langle\delta_{\mathrm{g}}^{2}\right\rangle^{2}$. We do not derive the moments $\left\langle\delta_{\mathrm{g}}^{2}\right\rangle$ and $\left\langle\delta_{\mathrm{g}}^{3}\right\rangle$ of the PDF by directly applying the computation scheme given in Eq. (15), but by correcting the count-in-cells statistics for discreteness effects using the Poissonian shot-noise model (e.g., Peebles 1980; Fry 1985, cf. Eqs. (374) and (375) of Bernardeau et al. 2002, possible biases introduced by this estimation technique are discussed by Hui \& Gaztañaga 1999). The corresponding values of $\sigma$ and $S_{3}$ for the local Universe (in redshift space) have been derived by Croton et al. 2004 using the $2 \mathrm{dFGRS}$. Here we plot the values corresponding to their $-21<\mathcal{M}-5 \log h<-20$ subsample, which actually brackets the median luminosity of our volume limited sample.

We can see that the rms amplitude of fluctuations of the VVDS density field, on scales $8 h^{-1} \mathrm{Mpc}$, is into a good approximation constant over the full redshift baseline investigated, with a mean value of $0.94 \pm 0.07$ over $0.7<z<1.5$.

While the strength of clustering of galaxies brighter than $\mathcal{M}<-20+5 \log h$ does not change much in this redshift interval, each VVDS measurement is lower than the value inferred at $z \sim 0$ by Croton et al. (2004). In particular, our mean value is $\sim 10 \%$ smaller than the $2 \mathrm{dFGRS}$ value and the difference is significant at $\sim 2 \sigma$ level.

The skewness $S_{3}$, which measures the tendency of gravitational clustering to create asymmetries between underdense and overdense regions, decreases as a function of redshift. We observe a systematic decrement not only internally to the VVDS sample, but also when we compare our measurements with the $z=0$ estimate. This trend is caused by the development of the low- $\delta$ tail in the PDF as a function of time on both the $R=8,10 h^{-1} \mathrm{Mpc}$ scales and reflects the fact that the probability of having underdense regions is greater at present epoch than it was at $z \sim 1.5$ (where its measured value is $\sim 2 \sigma$ lower.)

The amplitudes of the rms and skewness of galaxy overdensities show an evolutionary trend dissimilar from that predicted in first and second order perturbation theory for the gravitational growth of dark matter fluctuations (see Bernardeau et al. 2002 for a review). According to linear perturbation theory the amplitude of the rms of mass fluctuations scales with redshift as in Eq. (18) while second order perturbation theory predicts that, on the scales where the quasi-linear approximation holds, the growth rate of $\left\langle\delta_{\mathrm{g}}^{3}\right\rangle$ and variance $\left\langle\delta_{\mathrm{g}}^{2}\right\rangle^{2}$ are syncronized so that the skewness $S_{3}$ of an initially Gaussian fields should remain constant
(Peebles 1980; Juszkiewicz et al. 1993; Bernardeau 1993) ${ }^{1}$. Furthermore, in Le Fèvre et al. (2005c) we show that even the general shape of the galaxy PDF deviates from a lognormal distribution, i.e., from the profile in terms of which the mass PDF is generally approximated (see Sect. 5). Therefore, we conclude that the PDF evolution is not caused by gravity alone; the redshift scaling of its global shape and moments effectively indicates the presence of a time evolving bias.

We can deconvolve the purely gravitational signature and investigate properties and characteristics of the biasing between matter and galaxies by comparing the galaxy PDF to the corresponding statistics computed for mass fluctuations. Thus, we now turn to the problem of deriving the PDF of mass fluctuations.

\section{The PDF of mass fluctuations in redshift-distorted comoving coordinates}

The VVDS survey is providing a rich body of redshift data for mapping the galaxy density field in extended regions of space and over a wide interval of cosmic epochs. On the contrary, the direct determination of the underlying mass density field and its associated PDF is a less straightforward process. Nonetheless we may gain insight into the mass statistics by using simulations and theoretical arguments.

In the standard picture of gravitational instability, the PDF of the primordial cosmological mass density fluctuations is assumed to obey a random Gaussian distribution. Once the density fluctuations reach the non-linear stage, their PDF significantly deviates from the initial Gaussian profile and a variety of phenomenological models have been proposed to describe its shape (e.g., Saslaw 1985; Lahav et al. 1993). In particular, it is well established in CDM models that when structure formation has reached the nonlinear regime, the density contrasts in comoving space $f(\delta)$ follow, to a good approximation, a lognormal distribution (Coles \& Jones 1991; Kofman et al. 1994; Taylor \& Watts 2000; Kayo et al. 2001),

$f(\delta)=\frac{\left(2 \pi \omega^{2}\right)^{-1 / 2}}{1+\delta} \exp \left\{-\frac{\left[\ln (1+\delta)+\omega^{2} / 2\right]^{2}}{2 \omega^{2}}\right\}$.

This approximation becomes poor in the highly non-linear regime (e.g., Bernardeau \& Kofman 1995; Ueda \& Yokoyama 1996). The PDF of mass overdensities $f(\delta)$ is characterized by a single parameter $(\omega)$ that is related to the variance of the $\delta$ field as

$\omega^{2}=\ln \left[1+\left\langle\delta^{2}\right\rangle\right]$

At high redshifts, the variance $\sigma_{R}$ over sufficiently large scales $R$ (those explored in this paper) may be easily derived using the linear theory approximation:

$\sigma_{R}(z)=\sigma_{R}(z=0) D(z)$

where $D(z)$ is the linear growth rate of density fluctuations normalized to unity at $z=0$ (Heat 1977; Hamilton 2001).

\footnotetext{
${ }^{1}$ Note that the observed redshift evolution of the skewness is just the opposite of what is expected also in generic dimensional nonGaussian models where $S_{3}$ is predicted to increase with redshift.
} 
The lognormal approximation formally describes the distribution of matter fluctuations computed in real comoving coordinates. On the contrary, the PDF of galaxies is observationally derived in redshift space. In order to map properly the mass overdensities into galaxy overdensities the mass and galaxy PDFs must be computed in a common reference frame. It has been shown by Sigad et al. (2000) that an optimal strategy to derive galaxy biasing is to compare both mass and galaxy density fields directly in redshift space. Implicit in this approach is the assumption that mass and galaxies are statistically affected in the same way by gravitational perturbations, and thus, that there is no velocity bias in the motion of the two components.

A general model which allows the explicit computation of the statistical distortions caused by peculiar velocities has been proposed by Kaiser (1987). This applies in the linear regime (i.e., on large scales) and in the local Universe where redshift and distances are linearly related. At cosmological distances $\mathrm{z}$, however, the mapping between real comoving coordinates $(\boldsymbol{x})$ and redshift comoving coordinates $(\boldsymbol{y})$, i.e., the pseudo-comoving coordinates inferred on the basis of the observed redshifts, is less trivial, and we proceed to obtain it in the following.

In an inhomogeneous Universe, galaxies have motions above and beyond their Hubble velocity (e.g., Giovanelli et al. 1998; Marinoni et al. 1998; Branchini et al. 2001). As a consequence, Doppler spectral shifts add to the cosmological signal and the observed redshift $(\tilde{z})$ is given by

$\tilde{z}=z+\frac{U(\boldsymbol{x})}{c}(1+z)$

where $z$ is the cosmological redshift in a uniform FriedmanRobertson-Walker metric and where $U(\boldsymbol{x})=\boldsymbol{v}(\boldsymbol{x}) \cdot \hat{\boldsymbol{r}}=|\boldsymbol{v}(\boldsymbol{x})| \mu$ is the radial component of the peculiar velocity $(\mu$ is the cosine of the angle between the peculiar velocity vector and the lineof-sight versor $\hat{\boldsymbol{r}}$ ).

The redshift comoving distance of a galaxy at the observed redshift $\tilde{z}$ is thus

$y=\frac{c}{H_{0}} \int_{0}^{z+\frac{U}{c}(1+z)} \frac{1}{E(\chi)} \mathrm{d} \chi$,

where

$E(z)=\left[\Omega_{\mathrm{m}}(1+z)^{3}+\left(1-\Omega_{\mathrm{m}}-\Omega_{\Lambda}\right)(1+z)^{2}+\Omega_{\Lambda}\right]^{1 / 2}$.

At high redshifts $\left(z \gg \frac{|U|}{c}\right)$, we can write

$y=\frac{c}{H_{0}}\left[\int_{0}^{z} \frac{1}{E(\chi)} \mathrm{d} \chi+\frac{U}{c}(1+z) E(z)^{-1}\right]$

which, in turns, gives the coordinate transformation from real comoving space $\boldsymbol{x}$ to the redshift comoving space $\boldsymbol{y}$

$\boldsymbol{y}=\boldsymbol{x}\left[1+p(z) \frac{U(\boldsymbol{x})}{\boldsymbol{x}}\right]$

In this mapping, the cosmological term $p(z)$,

$p(z)=\frac{1+z}{H_{0} E(z)}$

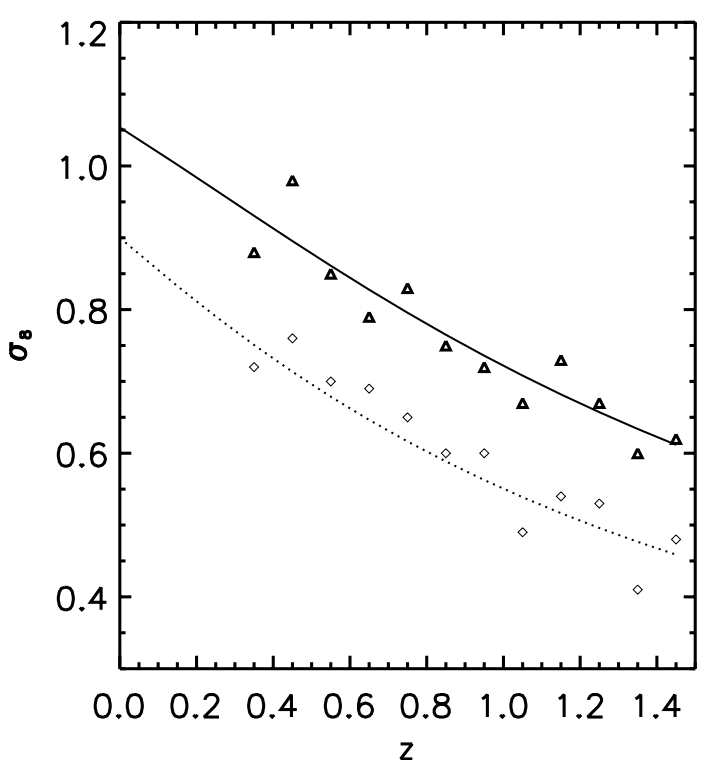

Fig. 8. Redshift scaling of the rms mass fluctuations in sphere of $8 h^{-1} \mathrm{Mpc}$ radius. Diamonds represent $\sigma_{8}$ as computed from the $\Lambda \mathrm{CDM}$ Hubble volume simulation in real comoving space ( $x$-space), while triangles represent the corresponding values recovered in the redshift perturbed comoving coordinates ( $y$-space). The solid line is the analytical prediction for the scaling of $\sigma_{8}$ in the $y$-space obtained using Eq. (30), while the dotted line represents the $x$-space evolution predicted in real space. In both cases the power spectrum of perturbations is the same and has been normalized in order to match the simulation specifications $\left(\sigma^{x}(z=0)=0.9\right)$.

is a correcting factor which takes into account the fact that, at high redshifts, distances do not scale linearly with redshift, and, thus, that peculiar velocities cannot be simply added to redshift space positions as in the local Universe.

The galaxy density field in the redshift-distorted space is related to the galaxy density in real space by the Jacobian of the transformation between the two coordinate systems

$\rho_{y}(\boldsymbol{y})=\rho_{x}(\boldsymbol{x})\left[1+p(z) \frac{U(\boldsymbol{x})}{\boldsymbol{x}}\right]^{-2}\left[1+p(z) \frac{\mathrm{d} U(\boldsymbol{x})}{\mathrm{d} \boldsymbol{x}}\right]^{-1}$.

At sufficiently large distances from the observer, neglecting the survey selection function (i.e., considering a volume-limited redshift survey) and at first order in perturbations we obtain

$\delta_{y}(\boldsymbol{y})=\delta_{x}(\boldsymbol{x})-p(z) \frac{\mathrm{d} U(\boldsymbol{x})}{\mathrm{d} \boldsymbol{x}}$.

The second term on the right hand side can be evaluated using linear-regime approximations and gravitational instability theory. In comoving coordinates it is given by

$\frac{\mathrm{d} U(\boldsymbol{x})}{\mathrm{d} \boldsymbol{x}}=-\frac{\mu^{2} f(z) H(z)}{1+z} \delta_{x}(\boldsymbol{x})$

where $f=\mathrm{d} \ln D / \mathrm{d} \ln a$ is the logarithmic derivative of the linear growth rate of density fluctuations with respect to the expansion factor $a(t)$. At redshift $z$ (corresponding to the comoving position $\boldsymbol{x}$ ) a useful approximation is given by:

$f(z) \sim \Omega_{m}^{3 / 5} E(z)^{-6 / 5}(1+z)^{9 / 5}$

(see Martel 1991; Lahav et al. 1991). 


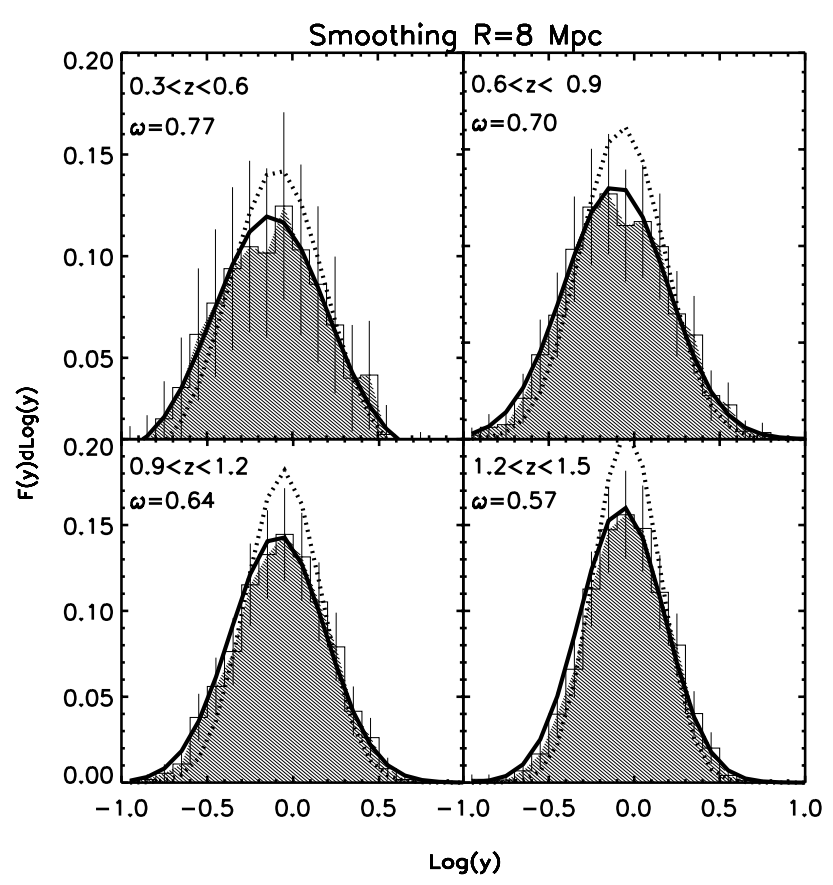

Fig. 9. One-point PDFs of dark matter fluctuations (shaded area) computed using the Hubble volume $\Lambda \mathrm{CDM}$ cosmological simulation in 4 different redshift ranges over a volume which mimics the geometry of the VVDS sample. The mass PDF has been recovered in the redshift comoving space by smoothing the mass-particle distribution with a TH window of size $R=8 h^{-1} \mathrm{Mpc}$. Note that the plotted histogram actually corresponds to $F(y)=(\ln 10) y f(y)$ because the binning $(\operatorname{dog} y=0.1)$ is done in $\log (y)=\log (1+\delta)$. The dotted line represents the lognormal approximation derived in the real comoving space using Eq. (16). The solid curve represents the lognormal approximation computed adopting the variance parameter $\omega$ (shown in the inset) theoretically inferred using Eq. (30), which models peculiar velocity distortions as a function of redshift.

By combining the previous results we obtain

$\delta_{y}(\boldsymbol{y})=\delta_{x}(\boldsymbol{x})\left[1+\mu^{2} f(z)\right]$.

which reduces to the Kaiser (1987) correction when $z=0$.

The relation between the azimuthally averaged variances measured in real and redshift comoving space is

$\sigma^{y}(z)=\left[1+\frac{2}{3} f(z)+\frac{1}{5} f^{2}(z)\right]^{1 / 2} \sigma^{x}(z)$.

We have tested the validity of Eq. (30) in the high redshift domain using the Hubble volume $N$-body simulations carried out by the Virgo consortium (Colberg et al. 2000). This is a large numerical experiment which allows the simulation of mass surveys along the observer past light cone. The simulated mass distribution is computed in a $\Lambda \mathrm{CDM}$ cosmogony with parameters $\Omega_{\mathrm{m}}=0.3, \Omega_{\Lambda}=0.7$ and $H_{0}=70 \mathrm{~km} \mathrm{~s}^{-1} \mathrm{Mpc}^{-1}$. The volume covered by this $N$-body simulation is large enough that the mass survey extracted along the diagonal of the simulation cubes extends up to the redshift of interest i.e., the redshift covered by the first-epoch VVDS data $(z=1.5)$. In this simulation the mass-particle resolution is $2.2 \times 10^{12} h^{-1} M_{\odot}$ and the present epoch is defined by a linear rms density fluctuation in a sphere of radius $8 h^{-1} \mathrm{Mpc}$ of $\sigma_{8}=0.9$.
The mass density contrasts in the redshift perturbed comoving coordinates $\delta\left(\boldsymbol{y}_{i}, R\right)$ have been calculated at random positions $\boldsymbol{y}_{i}$ in the simulation volume, by smoothing the particle distribution with a spherical top hat window of length $R=8 h^{-1} \mathrm{Mpc}$. Mass variances in different redshift bins are then derived using Eq. (15). The result is compared to the prediction of Eq. (30) in Fig. 8. Note that, even if it is clear that measurements suffer from cosmic variance due to the relatively small volume sampled at each redshift, the predictions of Eq. (30) are in agreement with the observed scaling of the linear mass variance. The magnitude of the correction with respect to the unperturbed case is also evident; mass fluctuations recovered in redshift space on a $8 h^{-1} \mathrm{Mpc}$ scale, in the redshift comoving coordinates, at $z=0.5(1.5)$ are $\sim 25(35) \%$ larger than in real comoving space (the correction factor is $\sim 17 \%$ in the local Universe). Figure 9 shows that this apparent enhancement in the rms fluctuations results in a broadening of the mass PDF recovered in the redshift comoving space. Thus, the effect of peculiar velocities is to shrink overdense regions and to inflate underdense regions, enhancing the probability of having large density fluctuations (both positive and negative).

We finally compare, in various redshift intervals, the accuracy with which the lognormal mass PDF derived in the redshift comoving space (by using Eq. (30) in 17) approximates the PDF directly inferred from the Hubble volume simulation (see Fig. 9). On a scale of $8 h^{-1} \mathrm{Mpc}$, the agreement between the analytical and simulated mass PDFs is satisfactory at all redshifts. This holds true also when the mass PDFs recovered on $R=5$ and $10 h^{-1} \mathrm{Mpc}$ scales are compared.

Thus, with a good degree of confidence, we can use Eq. (30) to predict the PDF of mass fluctuations in redshift distorted comoving coordinates (the same coordinates where the galaxy PDF is observed) and in a generic cosmological background. This allows us to speed up computation time and to frame the results about the biasing function in a generic cosmological model.

\section{Measuring galaxy biasing}

In this section we describe the method applied to determine the relationship between galaxy and mass overdensities. The galaxy overdensity field $\delta_{\mathrm{g}}$ depends in principle on various astrophysical and cosmological parameters such as spatial position $(r)$, underlying matter density fluctuations $(\delta)$, scale $R$ with which the density field is reconstructed, cosmological time $(z)$, galaxy colors, local gas temperature, non-local environment, etc.

For the purposes of this study, we will rely on the following simplifying theoretical assumptions:

i) the efficiency of galaxy formation on a given cosmological scale is sensitive only to the underlying mass distribution. This means that the galaxy fluctuation field is in a reasonably tight one-to-one relationship with the underlying mass fluctuation field, and that the biasing scheme may be formally represented via the relationship $\delta_{\mathrm{g}}=$ $b(z, \delta, R) \delta$. While such an approach represents a non-trivial step forward in understanding the properties of the biasing 
function $b$ (if compared, for example, to constant parameterizations of the biasing relation), it is however evident that the biasing function could show, in principle, a more complex functional dependence.

ii) The current theoretical understanding of how clustering of DM proceeds via gravitational instability in the expanding Universe is well developed, i.e., the PDF of mass fluctuations of the real Universe can be safely derived via analytical models or $N$-body simulations (see discussion in Sect. 6) In particular, in what follows, we will consider a $\Lambda \mathrm{CDM}$ background mass distribution locally normalized to $\sigma_{8}(z=0)=0.9$.

iii) The redshift distortions affect the densities of galaxies and mass in a similar way, i.e., there is no velocity bias between these two components, and galaxies follow the matter flow.

\subsection{The method}

As described in Sect. 1, we derive the relationship between galaxy and mass overdensities in redshift space $\delta_{\mathrm{g}}=\delta_{\mathrm{g}}(\delta)$ as the one-to-one transformation which maps the theoretical mass PDF $f(\delta)$ into the observed galaxy PDF $g\left(\delta_{\mathrm{g}}\right)$. A similar method to derive the biasing function has been proposed and tested using CDM simulations by Sigad et al. (2000) (see also Szapudi \& Pan 2004). This same technique has been recently applied in different contexts by Marinoni \& Hudson (2002) to derive the mass-to-light $(M=M(L))$ and the X-ray-to-optical ( $L_{x}=L_{x}(L)$ ) functions for a wide mass range of virialized systems, and by Ostriker et al. (2003) to explore the void phenomena in the context of hydrodynamic simulations.

Using Eq. (1)-(3), we obtain the biasing function $b(\delta)$ as the solution of the following differential equation

$$
\left\{\begin{array}{l}
\delta_{\mathrm{g}}(-1)=-1 \\
b^{\prime}(\delta) \delta+b(\delta)=f(\delta) g\left(\delta_{\mathrm{g}}\right)^{-1}
\end{array}\right.
$$

where the prime denotes the derivative with respect to $\delta, f(\delta)$ and $g(\delta)$ are the PDF of mass and galaxy fluctuations respectively, and the initial condition has been physically specified by requiring that galaxies cannot form where there is no mass.

With this approach, we loose information on a possible stochasticity characterizing the biasing function. The advantage is that we can provide a measure, on some characteristic scales $R$, of the local, non-linear, deterministic biasing function (Eq. (2)) over the continuous redshift interval $0.4<z<1.5$.

We have obtained the biasing function $b(\delta)$ by numerically integrating the differential Eq. (31), i) in different redshift intervals in order to follow the evolution of $b(\delta)$ as a function of cosmic time, and ii) using matter and galaxy PDFs obtained by smoothing the density fields on $R=5,8$, and $10 h^{-1} \mathrm{Mpc}$ in order to test the scale dependence of the galaxy biasing function.

The information contained in the non-linear function $b(\delta)$ can be compressed into a single scalar which may be easily compared to the constant values in term of which the biasing relation is usually parameterized (see Eq. (1)). Since, by definition, $\langle b(\delta) \delta\rangle=0$, the most interesting linear bias estimators are associated to the second order moments of the PDFs, i.e., the variance $\left\langle\delta_{\mathrm{g}}^{2}\right\rangle$ and the covariance $\left\langle\delta_{\mathrm{g}} \delta\right\rangle$. Following the prescriptions of Dekel \& Lahav (1999), we characterize the biasing function as follows:

$\hat{b} \equiv \frac{\left\langle b(\delta) \delta^{2}\right\rangle}{\left\langle\delta^{2}\right\rangle}$

and

$b_{\mathrm{L}}^{2} \equiv \frac{\left\langle b^{2}(\delta) \delta^{2}\right\rangle}{\left\langle\delta^{2}\right\rangle}$

where the parameter $\hat{b}$, measuring the slope of the linear regression of $\delta_{\mathrm{g}}$ on $\delta$, is the natural generalization of the linear bias parameter defined in equation 1 and $b_{\mathrm{L}}^{2}$ is an "unbiased estimator" of the linear biasing parameter defined as $\xi_{\mathrm{g}}=b^{2} \xi$, when the bias relation is deterministic, i.e., non-stochastic. The ratio $r=\hat{b} / b_{\mathrm{L}}$ is the relevant measure of nonlinearity in the biasing relation; it is unity for linear biasing, and it is either larger or smaller than unity for nonlinear biasing.

The errors in the measured values of the biasing parameter $b_{\mathrm{L}}$ have been computed using independent mock catalogs which implement all the selection functions of the VVDS. This allows us to incorporate in our error estimates the uncertainties due to cosmic variance.

\subsection{Testing the method}

Before applying the biasing computation scheme (Eq. (31)) to VVDS data, we have tested that the method can be meaningfully applied, i.e., it is free of systematics when implemented with samples of simulated galaxies which mimic all the observational systematics of our sample.

The procedure consists of computing the biasing function $\delta_{\mathrm{s}}=b\left(\delta_{\mathrm{p}}\right) \delta_{\mathrm{p}}$ between the density field $\delta_{\mathrm{s}}$ reconstructed using an s-sample (representing the pseudo-survey sample, see Sect. 4.1) and the density fluctuations $\delta_{\mathrm{p}}$ of the corresponding p-sample (representing the pseudo-real Universe). We have already determined the range of redshift, density contrasts and smoothing scales where the sample simulating all the VVDS selection functions (s-sample) trace the underlying density of galaxies ( $\mathrm{p}$-sample). We thus expect, for consistency, that, in that range, the biasing between the two samples derived by applying our computation scheme (Eq. (31), using the PDFs of the $\mathrm{s}$ - and p-samples) is independent of $\delta_{\mathrm{p}}$ and equal to $b\left(\delta_{\mathrm{p}}\right)=1$.

Results are presented in Fig. 10 for two different $\mathrm{TH}$ smoothing scales. Note that a log-log density plot is used in order to emphasize the behavior of the biasing function in underdense regions. We conclude that on scales $R \geq 8 h^{-1} \mathrm{Mpc}$ the density recovered by a "four-passes" VVDS-like survey is not biased with respect to the underlying distribution on any density scale and in any redshift interval up to $z=1.5$. As a matter of fact, the linear bias parameter with which information contained in the biasing function can be at first order approximated is $b_{\mathrm{L}} \sim 1$ and the biasing relation does not show any significant deviation from linearity as indicated by the fact that the $r$ parameter is also very close to unity.

If the density field is smoothed on $5 h^{-1} \mathrm{Mpc}$, the effects of the incompleteness in low-density regions (already discussed 

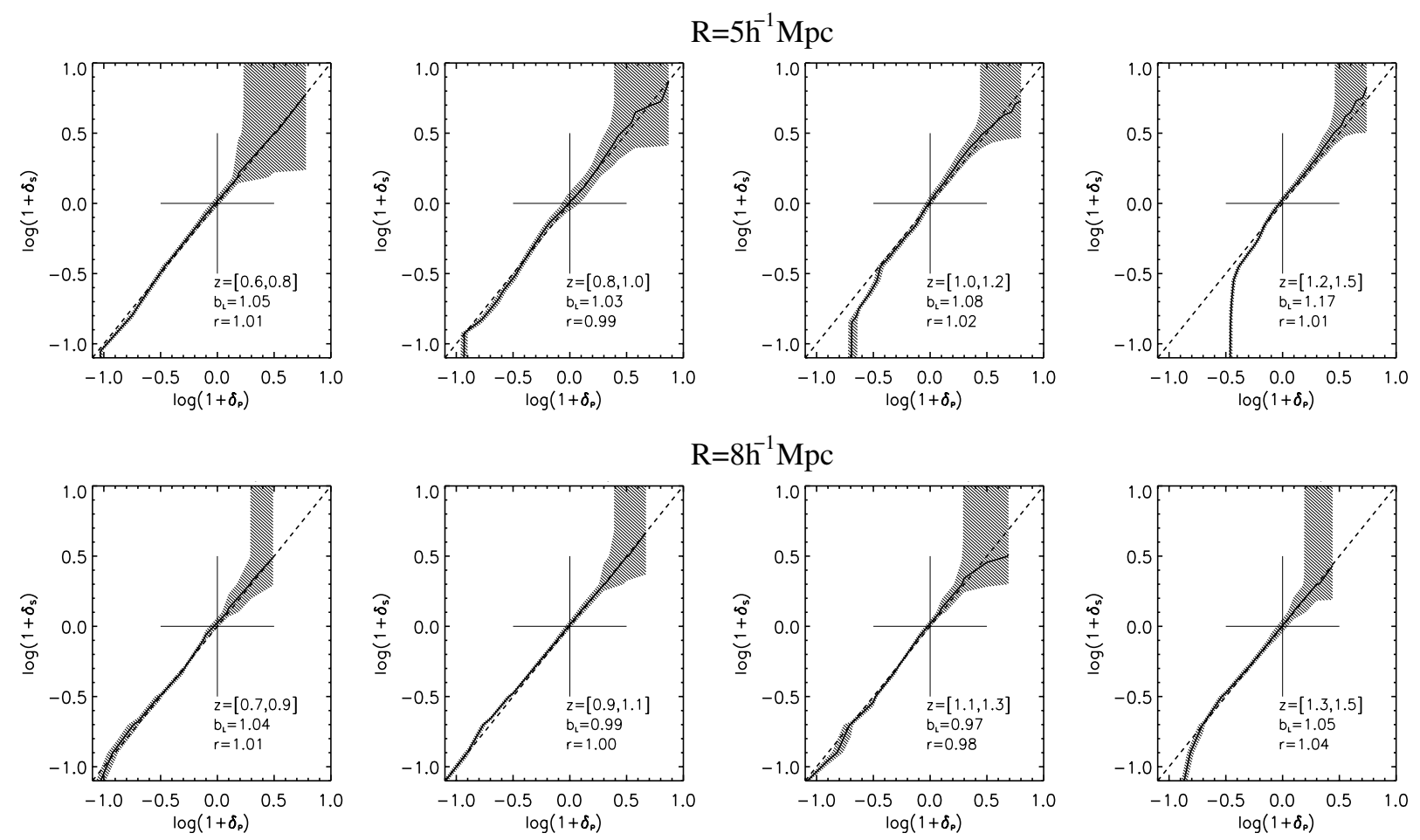

$\mathrm{R}=8 \mathrm{~h}^{-1} \mathrm{Mpc}$
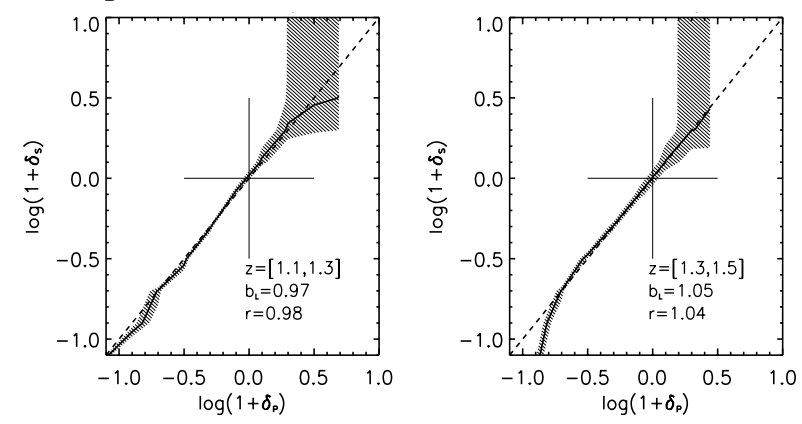

Fig. 10. The simulated biasing function (solid-line) at different cosmic epochs, between the density field traced by the s-sample (GALICS data simulating the VVDS sample, see Pollo et al. (2005) and Sect. 4.1) and the density field traced by the p-sample (GALICS data simulating the real underlying distribution of galaxies). $b_{\mathrm{L}}$ represent the linear bias parameter evaluated from the nonlinear biasing function using the estimator given in Eq. (32). The dashed line is drawn at $b_{\mathrm{L}}=1$ and represents the no bias case. The central cross is for reference and represents the $\delta_{\mathrm{g}}=\delta=0$ case. The $r$ parameter measures the deviations from the linearity. The galaxy overdensities are reconstructed using a TH window of sizes $R=5 h^{-1} \mathrm{Mpc}$ (upper panel) and $R=8 h^{-1} \mathrm{Mpc}$ (lower panel). The shadowed area represents $1 \sigma$ errors in the derived biasing function.

in Sect. 4.1, see Figs. 4 and 5) become evident. Underdense regions $\left(\log \left(1+\delta_{\mathrm{p}}\right) \lesssim-0.5\right)$ in volumes at redshift greater than 1 are poorly sampled with the VVDS survey strategy.

In the same spirit, we have also solved Eq. (31) to determine the biasing relation between the PDF of the Hubble volume mass fluctuations and the lognormal approximation given in Eq. (16). The biasing relation between these two different descriptions of the mass density field is linear and consistent with the no-bias hypothesis between the two representations of the density field on the scales we are interested in $\left(R \geq 5 h^{-1} \mathrm{Mpc}\right.$ and $\log (1+\delta)>-1)$.

\section{The biasing function up to $z \sim 1.5$}

\subsection{Results}

The numerical solutions of Eq. (31) for the $\mathcal{M}_{B}^{c}=-20+5 \log h$ volume-limited VVDS sample are plotted in various redshift slices, in Fig. 11 for the cases $R=8$ and $10 h^{-1} \mathrm{Mpc}$. Note that a log-log density plot is used in order to emphasize the behavior of the biasing function in underdense regions (note that in these units linear biasing appears as a curved line).

The corresponding parameters $b_{\mathrm{L}}$ and $r$ (also computed for the whole flux-limited sample) are quoted in Table 1, together with our estimates of the second moment of the galaxy PDF $\sigma_{R}$ and of the skewness parameter $\mathrm{S}_{3}$. Both these statistics have been computed as described in Sect. 5. Also note that the values of $\sigma_{8}$ measured for the flux-limited sample are consistent with the values independently derived in Sect. 3.4 on the basis of the results of the analysis of the clustering properties of VVDS galaxies (Paper III).

An empirical fit of the biasing function is obtained by using a formula similar to the one proposed by Dekel \& Lahav (1999)

$\delta_{\mathrm{g}}(\delta)= \begin{cases}\left(1+a_{0}\right)(1+\delta)^{a_{1}}-1 & \delta \leq 0 \\ a_{0}+a_{2} \delta+a_{3} \delta^{2} & \delta>0\end{cases}$

which best describes the behavior of biasing in underdense regions $(\delta<0)$, either the second order Taylor expansion of the density contrast of dark matter (Fry\& Gaztañaga 1993)

$\delta_{\mathrm{g}}=\sum_{k=0}^{2} \frac{b_{k}}{k !} \delta^{k}$

which allows an easier comparison of our results with other studies. The best fitting parameters of these non-linear approximations are quoted in Table 2.

The dependence of the shape of the biasing function on galaxy luminosity is plotted in Fig. 12. Results are shown at the median depth of the VVDS sample (in the redshift bin $0.7<z<0.9)$ where faint objects $\left(\mathcal{M}_{B}<-17.7+5 \log h\right)$ are still sampled.

In Fig. 13 we show the redshift evolution of the linear biasing parameter $b_{\mathrm{L}}$ computed over the redshift interval $0.4<$ $z<1.5$ for both the flux and volume limited samples. We can conclude that biasing is not changing with cosmic time for 
Table 1. Bias measurements from the VVDS first epoch data.

\begin{tabular}{|c|c|c|c|c|c|c|c|}
\hline $\begin{array}{l}R \\
h^{-1} \mathrm{Mpc}\end{array}$ & Redshift range & $\overline{\mathcal{M}_{B}^{c}}$ & $\bar{~} N_{\text {gal }}$ & $b_{\mathrm{L}}$ & $\bar{r}$ & $\overline{\sigma_{R}}$ & $\overline{S_{3}}$ \\
\hline \multirow[t]{3}{*}{5} & $0.4<z<0.7$ & No & 1583 & $0.87 \pm 0.15$ & 0.95 & $0.94 \pm 0.15$ & $1.2 \pm 0.3$ \\
\hline & $0.7<z<0.9$ & & 1044 & $0.95 \pm 0.15$ & 0.96 & $0.98 \pm 0.15$ & $1.4 \pm 0.3$ \\
\hline & $0.9<z<1.1$ & & 759 & $0.97 \pm 0.13$ & 0.97 & $0.93 \pm 0.13$ & $1.1 \pm 0.3$ \\
\hline \multirow[t]{3}{*}{5} & $0.4<z<0.7$ & -18.7 & 610 & $1.06 \pm 0.17$ & 0.97 & $1.18 \pm 0.17$ & $1.6 \pm 0.3$ \\
\hline & $0.7<z<0.9$ & & 726 & $1.03 \pm 0.15$ & 0.97 & $1.05 \pm 0.15$ & $1.6 \pm 0.3$ \\
\hline & $0.9<z<1.1$ & & 751 & $1.00 \pm 0.14$ & 0.97 & $0.97 \pm 0.14$ & $1.5 \pm 0.3$ \\
\hline \multirow[t]{3}{*}{5} & $0.4<z<0.7$ & -20 & 160 & $1.10 \pm 0.18$ & 0.97 & $1.28 \pm 0.18$ & $1.4 \pm 0.4$ \\
\hline & $0.7<z<0.9$ & & 229 & $1.12 \pm 0.17$ & 0.99 & $1.18 \pm 0.17$ & $1.1 \pm 0.3$ \\
\hline & $0.9<z<1.1$ & & 289 & $1.18 \pm 0.15$ & 0.97 & $1.17 \pm 0.15$ & $1.4 \pm 0.4$ \\
\hline \multirow[t]{4}{*}{8} & $0.7<z<0.9$ & No & 1263 & $0.92 \pm 0.20$ & 0.97 & $0.67 \pm 0.20$ & $1.6 \pm 0.4$ \\
\hline & $0.9<z<1.1$ & & 864 & $1.03 \pm 0.16$ & 0.96 & $0.74 \pm 0.16$ & $1.6 \pm 0.3$ \\
\hline & $1.1<z<1.3$ & & 440 & $1.21 \pm 0.12$ & 0.96 & $0.82 \pm 0.12$ & $1.3 \pm 0.3$ \\
\hline & $1.3<z<1.5$ & & 234 & $1.51 \pm 0.10$ & 0.95 & $0.96 \pm 0.11$ & $1.0 \pm 0.3$ \\
\hline \multirow[t]{2}{*}{8} & $0.7<z<0.9$ & -18.7 & 879 & $0.98 \pm 0.21$ & 0.98 & $0.75 \pm 0.21$ & $1.6 \pm 0.4$ \\
\hline & $0.9<z<1.1$ & & 813 & $1.01 \pm 0.16$ & 0.97 & $0.72 \pm 0.16$ & $1.8 \pm 0.3$ \\
\hline \multirow[t]{4}{*}{8} & $0.7<z<0.9$ & -20 & 279 & $1.17 \pm 0.23$ & 0.98 & $0.89 \pm 0.23$ & $1.7 \pm 0.4$ \\
\hline & $0.9<z<1.1$ & & 327 & $1.30 \pm 0.17$ & 0.99 & $0.94 \pm 0.17$ & $1.8 \pm 0.3$ \\
\hline & $1.1<z<1.3$ & & 251 & $1.33 \pm 0.12$ & 0.96 & $0.88 \pm 0.12$ & $1.2 \pm 0.3$ \\
\hline & $1.3<z<1.5$ & & 169 & $1.56 \pm 0.11$ & 0.95 & $0.99 \pm 0.11$ & $1.1 \pm 0.3$ \\
\hline \multirow[t]{4}{*}{10} & $0.7<z<0.9$ & No & 1425 & $1.03 \pm 0.22$ & 0.91 & $0.66 \pm 0.22$ & $1.6 \pm 0.4$ \\
\hline & $0.9<z<1.1$ & & 955 & $1.05 \pm 0.18$ & 0.97 & $0.64 \pm 0.18$ & $1.7 \pm 0.4$ \\
\hline & $1.1<z<1.3$ & & 480 & $1.17 \pm 0.13$ & 0.90 & $0.68 \pm 0.13$ & $1.3 \pm 0.3$ \\
\hline & $1.3<z<1.5$ & & 250 & $1.55 \pm 0.11$ & 0.93 & $0.84 \pm 0.13$ & $1.2 \pm 0.3$ \\
\hline \multirow[t]{2}{*}{10} & $0.7<z<0.9$ & $\begin{array}{l}-18.7 \\
\end{array}$ & 991 & $1.03 \pm 0.25$ & 0.95 & $0.69 \pm 0.25$ & $1.5 \pm 0.4$ \\
\hline & $0.9<z<1.1$ & & 900 & $1.03 \pm 0.18$ & 0.96 & $0.63 \pm 0.18$ & $1.7 \pm 0.3$ \\
\hline \multirow[t]{4}{*}{10} & $0.7<z<0.9$ & -20 & 316 & $1.14 \pm 0.25$ & 0.92 & $0.75 \pm 0.25$ & $1.6 \pm 0.4$ \\
\hline & $0.9<z<1.1$ & & 360 & $1.26 \pm 0.20$ & 0.97 & $0.78 \pm 0.20$ & $1.8 \pm 0.4$ \\
\hline & $1.1<z<1.3$ & & 266 & $1.36 \pm 0.14$ & 0.91 & $0.78 \pm 0.14$ & $1.3 \pm 0.3$ \\
\hline & $1.3<z<1.5$ & & 175 & $1.54 \pm 0.13$ & 0.93 & $0.84 \pm 0.13$ & $1.3 \pm 0.3$ \\
\hline
\end{tabular}

Table 2. Best fitting parameters of the non linear biasing models given in Eqs. (34) and (35). Errors do not include cosmic variance.

\begin{tabular}{|c|c|c|c|c|c|c|c|c|c|}
\hline $\begin{array}{l}R \\
h^{-1} \mathrm{Mpc}\end{array}$ & $\begin{array}{c}\text { Redshift } \\
\text { range }\end{array}$ & $\overline{M_{B}^{c}}$ & $\overline{a_{0}}$ & $\overline{a_{1}}$ & $\overline{a_{2}}$ & $\overline{a_{3}}$ & $\overline{b_{0}}$ & $\overline{b_{1}}$ & $b_{2}$ \\
\hline \multirow[t]{4}{*}{8} & $0.7<z<0.9$ & No & $0.36 \pm 0.03$ & $1.36 \pm 0.11$ & $0.60 \pm 0.12$ & $-0.01 \pm 0.02$ & $0.10 \pm 0.06$ & $0.88 \pm 0.10$ & $-0.12 \pm 0.08$ \\
\hline & $0.9<z<1.1$ & & $0.35 \pm 0.03$ & $1.36 \pm 0.40$ & $1.08 \pm 0.10$ & $-0.08 \pm 0.02$ & $0.23 \pm 0.06$ & $1.18 \pm 0.09$ & $-0.20 \pm 0.06$ \\
\hline & $1.1<z<1.3$ & & $0.39 \pm 0.04$ & $1.64 \pm 0.13$ & $1.14 \pm 0.11$ & $-0.08 \pm 0.02$ & $0.24 \pm 0.07$ & $1.26 \pm 0.10$ & $-0.22 \pm 0.06$ \\
\hline & $1.3<z<1.5$ & & $0.54 \pm 0.05$ & $2.34 \pm 0.30$ & $1.46 \pm 0.14$ & $-0.11 \pm 0.02$ & $0.37 \pm 0.12$ & $1.57 \pm 0.15$ & $-0.24 \pm 0.08$ \\
\hline \multirow[t]{4}{*}{8} & $0.7<z<0.9$ & -20 & $0.25 \pm 0.05$ & $1.67 \pm 0.20$ & $1.25 \pm 0.16$ & $-0.09 \pm 0.03$ & $0.18 \pm 0.10$ & $1.30 \pm 0.15$ & $-0.20 \pm 0.08$ \\
\hline & $0.9<z<1.1$ & & $0.40 \pm 0.06$ & $2.12 \pm 0.33$ & $1.20 \pm 0.16$ & $-0.05 \pm 0.02$ & $0.23 \pm 0.17$ & $1.29 \pm 0.16$ & $-0.12 \pm 0.08$ \\
\hline & $1.1<z<1.3$ & & $0.45 \pm 0.04$ & $1.86 \pm 0.13$ & $1.29 \pm 0.13$ & $-0.11 \pm 0.02$ & $0.31 \pm 0.08$ & $1.40 \pm 0.13$ & $-0.26 \pm 0.06$ \\
\hline & $1.3<z<1.5$ & & $0.48 \pm 0.05$ & $2.51 \pm 0.30$ & $1.46 \pm 0.16$ & $-0.10 \pm 0.02$ & $0.33 \pm 0.14$ & $1.55 \pm 0.15$ & $-0.22 \pm 0.08$ \\
\hline \multirow[t]{4}{*}{10} & $0.7<z<0.9$ & No & $0.25 \pm 0.03$ & $0.85 \pm 0.10$ & $1.44 \pm 0.21$ & $-0.23 \pm 0.06$ & $0.17 \pm 0.07$ & $1.20 \pm 0.15$ & $-0.30 \pm 0.12$ \\
\hline & $0.9<z<1.1$ & & $0.18 \pm 0.02$ & $1.20 \pm 0.08$ & $1.23 \pm 0.13$ & $-0.18 \pm 0.04$ & $0.20 \pm 0.04$ & $1.19 \pm 0.09$ & $-0.34 \pm 0.08$ \\
\hline & $1.1<z<1.3$ & & $0.39 \pm 0.04$ & $1.30 \pm 0.11$ & $1.04 \pm 0.13$ & $-0.08 \pm 0.03$ & $0.26 \pm 0.06$ & $1.18 \pm 0.10$ & $-0.22 \pm 0.08$ \\
\hline & $1.3<z<1.5$ & & $0.54 \pm 0.04$ & $2.09 \pm 0.13$ & $1.41 \pm 0.15$ & $-0.14 \pm 0.03$ & $0.39 \pm 0.08$ & $1.55 \pm 0.12$ & $-0.32 \pm 0.08$ \\
\hline \multirow[t]{4}{*}{10} & $0.7<z<0.9$ & -20 & $0.21 \pm 0.03$ & $1.33 \pm 0.10$ & $1.45 \pm 0.16$ & $-0.22 \pm 0.04$ & $0.18 \pm 0.06$ & $1.26 \pm 0.11$ & $-0.28 \pm 0.10$ \\
\hline & $0.9<z<1.1$ & & $0.18 \pm 0.04$ & $1.51 \pm 0.15$ & $1.38 \pm 0.16$ & $-0.13 \pm 0.03$ & $0.20 \pm 0.08$ & $1.36 \pm 0.14$ & $-0.26 \pm 0.08$ \\
\hline & $1.1<z<1.3$ & & $0.38 \pm 0.04$ & $1.37 \pm 0.17$ & $1.50 \pm 0.15$ & $-0.13 \pm 0.03$ & $0.37 \pm 0.10$ & $1.50 \pm 0.14$ & $-0.26 \pm 0.08$ \\
\hline & $1.3<z<1.5$ & & $0.22 \pm 0.05$ & $1.76 \pm 0.20$ & $1.83 \pm 0.16$ & $-0.18 \pm 0.03$ & $0.33 \pm 0.12$ & $1.73 \pm 0.17$ & $-0.34 \pm 0.08$ \\
\hline
\end{tabular}

$z<0.8$, while there is a more pronounced evolution of biasing in the redshift interval $[0.8,1.5]$. In particular, the difference between the value of $b_{\mathrm{L}}$ at redshift $z \sim 1.5$ and $z \sim 0$ for a population of galaxies with luminosity $\mathcal{M}_{B}<-20+5 \log h$ is $\Delta b_{\mathrm{L}} \sim 0.5 \pm 0.14$, thus significant at a confidence level greater than $3 \sigma$.
In Fig. 14 we show the dependence of the linear biasing parameter on galaxy luminosity. Intrinsically brighter galaxies are more strongly biased than less luminous ones at every redshift and the dependence of biasing on luminosity at $z \sim 0.8$ is in good agreement with what is observed in the local Universe (Norberg et al. 2001). 
Given the difference in the rest-frame colors of elliptical and irregular galaxies and the fact that the observed $I$ band corresponds to bluer rest-frame bands at higher redshift, the relative fraction of early- and late-type galaxies in our $I$ band limited survey will change as a function of redshift.

Specifically, the observed difference in the $B$-band luminosity function of early- and late-types (Zucca et al. 2005), implies that the VVDS survey selects preferentially late-type galaxies at higher redshift. It is known that at $z=0$ late-type galaxies cluster less strongly than early-types (e.g., Giovanelli et al. 1986; Guzzo et al. 1997; Giuricin et al. 2001; Madgwick et al. 2002; Zehavi et al. 2002), and, thus, we might observe a variation of the amplitude of density fluctuations at high redshifts just because the morphological composition of our sample changes.

In order to disentangle the spurious morphological contribution to the observed evolution of the global biasing function we have splitted our sample according to rest frame colors, selecting a red $\left((B-I)_{0}>0.95 ; 849\right.$ galaxies in the 4-passes region with $z>0.7)$ and a blue subsample of galaxies $\left((B-I)_{0}<0.68 ; 1891\right.$ galaxies with $\left.z>0.7\right)$. These color cuts roughly correspond to selecting, respectively, morphological types $\leq I I$ and IV according to the classification scheme devised by Zucca et al. 2005 for the VVDS sample.

Clearly, this subsample selection does not correspond to the ideal case of a redshift survey sampling galaxies according to their rest-frame colors; however, useful information about differences in clustering between red and blue populations can still be inferred.

Note that the hypothesis on which the technique of comparing mass and galaxy density distributions is based (Sect. 6) can be straightforwardly generalized to compute the biasing between the density distributions of different galaxy types. In particular, we assume that the large scale velocities of late and early types are not dissimilar relative to each other (as it is effectively observed at $z=0$ e.g., Dekel 1994; Marinoni et al. 1998) i.e., the two velocity fields are noisy versions of the same underlying field.

Results about the color dependence of biasing are summarized in Table 3 and graphically presented in Fig. 15. The red sample is systematically a more biased tracer of mass than the blue one in every redshift interval investigated (i.e., $b^{r}>b^{b}$ ), but the relative biasing between the two populations is nearly constant $\left(b^{r} / b^{b} \sim 1.4 \pm 0.1\right)$

\subsection{Analysis and discussion}

\subsubsection{Biasing for the global galaxy population}

Here we examine and interpret the results derived in the previous section. We begin by discussing the general shape of the non-linear biasing function, for the global population, in different density regions. Our results can be summarized as follows:

i) in underdense regions $(1+\delta<1)$ the local slope of the biasing function $b(\delta)$ is always larger than unity even when the global slope is $b_{\mathrm{L}}<1$ (see for example Fig. 12). The fact that galaxies in low-mass density regions are always positively biased with respect to the mass distribution (i.e., locally $b>1$ ) is possibly physically caused by the fact that galaxies do not form in very low-density mass regions, i.e., below some finite mass underdensity the galaxy formation efficiency drops to zero. Using the biasing relation given in Eq. (34) the characteristic mass density threshold $\delta_{\mathrm{c}}$ below which very few galaxies form $\left(\delta_{\mathrm{g}} \leq-0.9\right)$, can be approximated as

$\log \left(1+\delta_{\mathrm{c}}\right) \sim-\frac{1+\log \left(1+a_{0}\right)}{a_{1}}$

There is evidence that this mass-density threshold, characterizing regions avoided by galaxies, increases as a function of redshift (see Fig. 11) and luminosity (see Fig. 12). If we consider $R=8 h^{-1} \mathrm{Mpc}$ and the redshift bin $0.7<z<0.9$ we see that while faint galaxies seem to be present even where the mass density contrast is very low (left panel of Fig. 12, $\left.\log \left(1+\delta_{\mathrm{c}}\right)=-0.96 \pm 0.09\right)$,

brighter galaxies do not seem to form in deep mass underdensities (right panel of Fig. 12, $\log \left(1+\delta_{\mathrm{c}}\right)=-0.73 \pm 0.11$ ). Therefore low-density regions are preferentially inhabited by low luminosity galaxies.

Moreover the mass-density threshold below which the formation of bright galaxies $\left(\mathcal{M}_{B}<-20+5 \log h\right)$ seems to be inhibited increases, irrespective of the scale investigated (see Fig. 11) as a function of redshift. On a scale $R=8 h^{-1} \mathrm{Mpc}$, the threshold shifts from $\log \left(1+\delta_{\mathrm{c}}\right)=-0.73 \pm 0.10$ at $z=0.8$ to $\log \left(1+\delta_{\mathrm{c}}\right) \sim-0.55 \pm 0.07$ at $z=1.4$ This suggests that galaxies of a given luminosity were tracing systematically higher mass overdensities in the early Universe, i.e, as time progresses, galaxy formation begins to take place also in lower density peaks.

ii) Even in regions where the mass density distribution is close to its mean value $(1+\delta \sim 1)$ bright galaxies are not unbiased tracer of the mass-overdensity field (Fig. 11). This can also be seen by setting $\delta=0$ in Eq. (34) and noting that $\delta_{\mathrm{g}}(\delta=0)=a_{0}>0$ for both analyzed samples (flux- and volume-limited) in all redshift ranges (see Table 2). This result is at variance with what is expected within the simple linear biasing picture, where, by construction, $\delta_{\mathrm{g}}(\delta=0)=0$.

iii) In higher matter-density environments $(1+\delta>1)$ galaxies were progressively more biased mass tracers in the past, i.e., the local slope $b(\delta)$ systematically increases with redshift on every scale investigated (Fig. 11). There is some indication that, at the upper tail of the mass density distribution, galaxies are anti-biased with respect to mass on all scales (i.e., the local slope is $b(\delta)<1$ for $\delta \gg 1$ ). Antibiasing in overdense regimes is a feature actually observed in simulations (e.g., Sigad et al. 2000; Somerville et al. 2001) and expected in theoretical models (e.g., Taruya \& Sato 2000). Physically this could be due to the merging of galaxies which reduces the number density of visible objects in high density regions or because galaxy formation is inhibited in regions where the gas is too hot to collapse and form stars.

iv) In general the linear approximation offers a poor description of the richness of details encoded in the biasing function. As a matter of fact the linear biasing function (dotted line in Figs. 11 and 12) poorly describes, in many cases, the observed scaling of the biasing relation (solid line). At the comoving scales of $R=5,8$ and $10 h^{-1} \mathrm{Mpc}$, non-linearities in the 

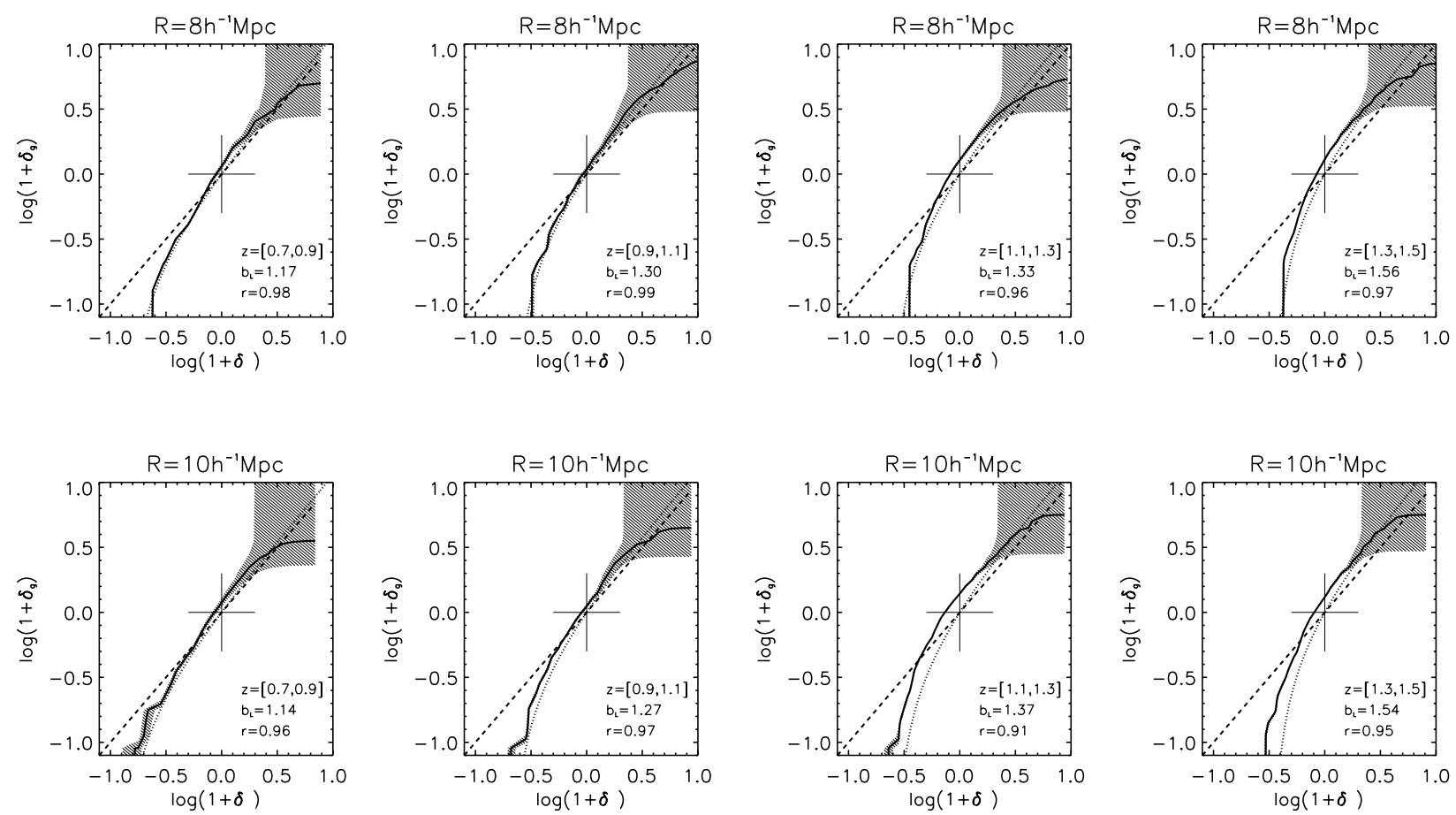

Fig. 11. The observed biasing function (solid-line) recovered for the density field smoothed on scales $R=8 h^{-1} \mathrm{Mpc}$ (upper panel) and $10 h^{-1} \mathrm{Mpc}$ (lower panel) and for different redshift bins (from left to right) in the volume-limited VVDS sample $\left(\mathcal{M}_{B}^{c}=-20+5 \log h\right)$. The dotted line represents the linear biasing model $\delta_{\mathrm{g}}=b_{\mathrm{L}} \delta$ while the no-bias case $\left(b_{\mathrm{L}}=1\right)$ is shown with a dashed line. The central cross is for reference and represents the $\delta_{\mathrm{g}}=\delta=0$ case. The shaded area represents $1 \sigma$ errors in the derived biasing function. Errors take into account the noise in the observed galaxy $\operatorname{PDF}\left(g\left(\delta_{\mathrm{g}}\right)\right)$, but do not include uncertainties due to cosmic variance.
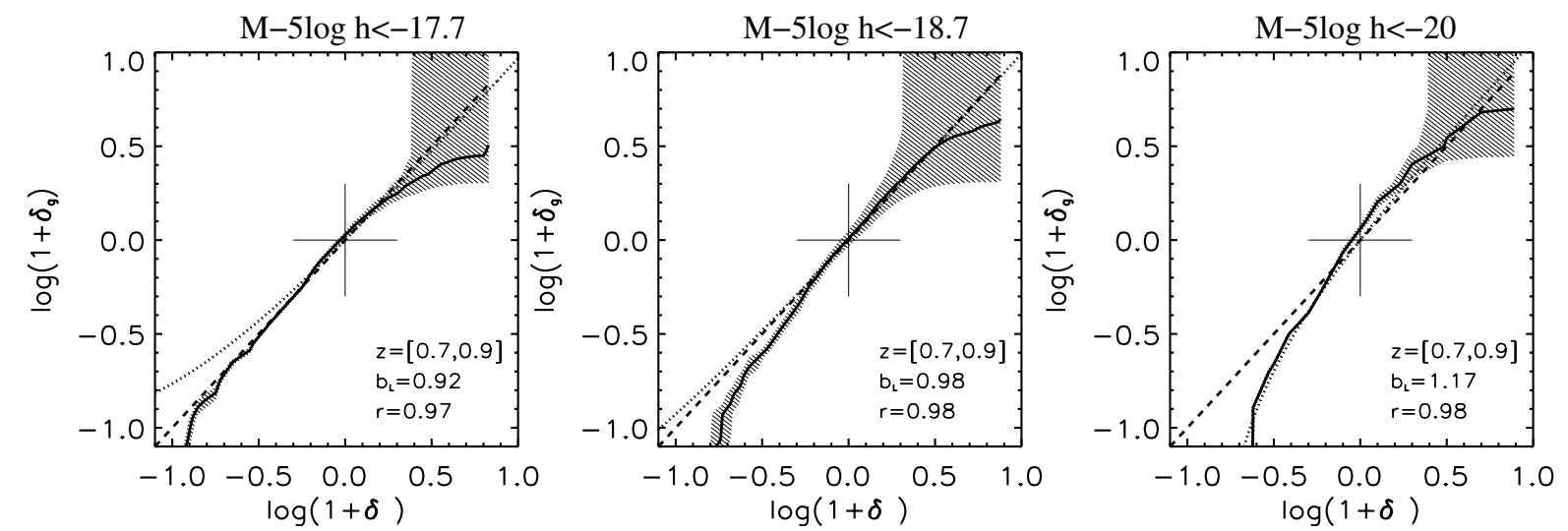

Fig. 12. The biasing function (solid-line) on scales $R=8 h^{-1} \mathrm{Mpc}$ and in the redshift interval $0.7<z<0.9$ computed for different luminosity classes. Symbols are as in Fig. 11.

biasing relation are typically $(1-r) \lesssim 10 \%$ in the redshift range investigated. We find that the ratio $b_{2} / b_{1}$ between the quadratic and linear term of the series approximation given in Eq. (35) is nearly constant in the redshift range $0.7<z<1.5$ and does not depend on luminosity (i.e. it is nearly the same for the fluxand volume-limited subsamples) or smoothing scale. We find that, on average, $b_{2} / b_{1} \sim-0.15 \pm 0.04$ for $R=8 h^{-1} \mathrm{Mpc}$ and $b_{2} / b_{1} \sim-0.19 \pm 0.04$ for $R=10 h^{-1} \mathrm{Mpc}$.

To facilitate comparison with other studies, which generally focus on the linear representation of biasing, we now discuss the properties of the linear approximation of our biasing function. The general characteristics of the linear parameter $b_{\mathrm{L}}$ can be summarized as follows:

v) by inspecting Table 1, we do not find any significant evidence that the global value of the linear biasing parameter $b_{\mathrm{L}}$ depends on the smoothing scale. Any possible systematic variation, if present, is smaller than the amplitude of our errorbars $(\sim 0.15)$. This scale independence in the biasing relation extends into the high redshift regimes similar conclusions obtained in the local Universe by the 2dFGRS on scales $>5 h^{-1} \mathrm{Mpc}$ (Verde et al. 2002). Moreover our results may be interpreted as a supporting evidence for theoretical arguments suggesting that bias is expected to be scale-independent on scales larger than a few $h^{-1}$ Mpc (e.g., Mann et al. 1998; Weinberg et al. 2004).

Since we find no evidence of scale-dependent bias, and since with different $R$ scales we are probing different redshift regimes, in Fig. 13 we have averaged the linear biasing parameters measured on 5, 8, and $10 h^{-1} \mathrm{Mpc}$ scales (values quoted in Table 1) in order to follow, in a continuous way, the 


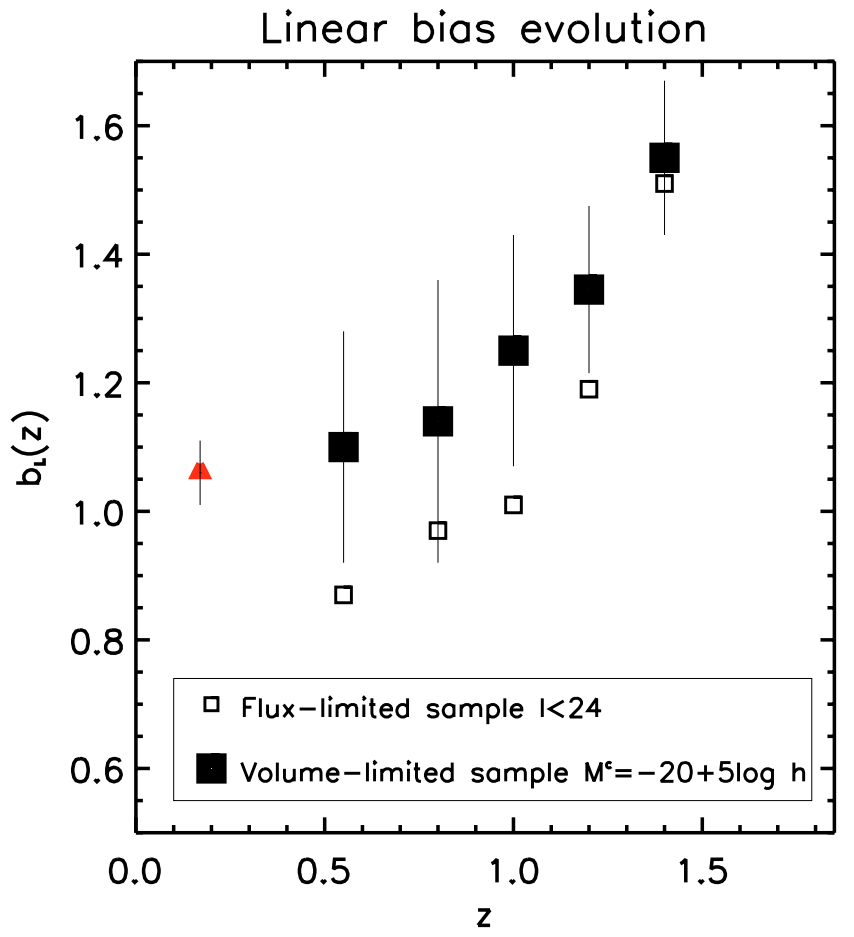

Fig. 13. The redshift evolution of the linear biasing parameter $b_{\mathrm{L}}$ for the volume-limited $\left(\mathcal{M}_{B}^{c}<-20+5 \log h\right)$ subsample (filled squares) is compared to the evolution of the biasing parameter for the whole fluxlimited VVDS-02h sample (empty squares). Since there is no significant evidence of scale dependence in the biasing relation, we have averaged the biasing parameters measured on 5,8, and $10 \mathrm{~h}^{-1} \mathrm{Mpc}$ scales in order to cover the full redshift baseline $0.4<z<1.5$. For clarity, only the errorbars corresponding to the volume-limited sample are shown. The triangle represents the $z \sim 0$ bias inferred for 2dFGRS galaxies having median $L / L^{*} \sim 2$ (i.e., the median luminosity of the volume-limited VVDS sample) as explained in the text.

redshift evolution of the linear galaxy biasing over the larger redshift baseline $0.4<z<1.5$. Figure 13 shows that $b_{\mathrm{L}}$ for galaxies brighter than $\mathcal{M}_{B}=-20+5 \log h$ changes from $1.10 \pm 0.18$ at $z \sim 0.55$ to $1.55 \pm 0.12$ at $z \sim 1.4$.

An even steeper variation is observed for the biasing of the flux-limited sample, indicating that biasing depends on galaxy luminosity. Figure 13 shows that the ratio between the amplitude of galaxy fluctuations and the underlying mass fluctuations declines with cosmic time. This scaling is effectively predicted within the framework of the peaks-biasing theoretical model (Kaiser 1984). At early times, galaxies are expected to form at the highest peaks of the density field since one needs a dense enough clump of baryons in order to start forming stars. Such high- $\sigma$ peaks are highly biased tracers of the underlying mass density field. According to this picture, as time progresses and the density field evolves, galaxy formation moves to lower$\sigma$ peaks, nonlinear peaks become less rare events and thus galaxies become less biased tracers of the mass density field. Additional "debiasing" mechanisms may contribute to the observed scaling shown in Fig. 13. It is likely that the densest regions stop forming new galaxies because their gas becomes too hot, cannot cool efficiently, and thus cannot collapse and form stars (Blanton et al. 1999). As galaxy formation moves

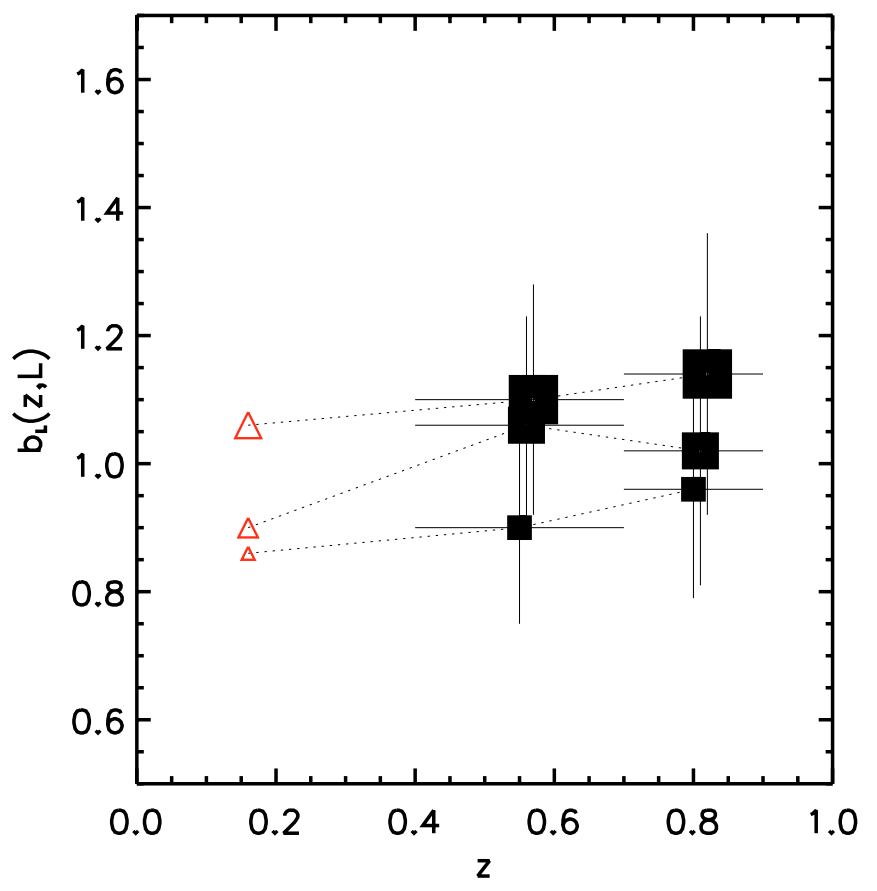

Fig. 14. Comparison between the galaxy linear bias parameter measured in the redshift interval $0.4<z<0.9$ for 3 different luminosity classes (squares) and the corresponding local estimates provided by the 2dFGRS (triangles). Points with increasing sizes correspond to three different volume-limited VVDS subsamples, i.e $\mathcal{M}_{B}-5 \log h<$ $-17.7,<-18.7$ and $<-20$, respectively. For clarity, squares with increasing size have been progressively displaced rightward to avoid crowding. The $z \sim 0$ measurements have been interpolated by using the formula describing the luminosity dependence of the 2dFGRS bias parameter (Norberg et al. 2001), the bias parameter for the 2dFGRS $L^{*}$ sample (i.e., $b_{*}=0.92$ (Verde et al. 2002)) and the median luminosity of the three VVDS subsamples $\left(L / L^{*}=0.52,0.82,2.0\right.$ respectively).

out of the hottest (and rarest) regions of the Universe, the biasing decreases. Finally, we also note that in order to derive the biasing function we have assumed that there is no difference in the velocity field of the luminous and matter components. After galaxies form, they are subject to the same gravitational forces as the dark matter, and thus they tend to trace the dark matter distribution more closely with time as shown by Dekel \& Rees (1987); Fry (1996); Tegmark \& Peebles (1998).

vi) In Fig. 13 we also show, for comparison, the value of the 2dFGRS linear biasing parameter inferred at $z=0.17$ (the effective depth of the survey) as the ratio between the $\sigma_{8}$ value measured by Croton et al. 2004 (in redshift-distorted space; see their Figs. 3 and 4) for a sample of objects with $-21<\mathcal{M}_{B}-5 \log h<-20$ (which actually brackets the median luminosity of our volume limited sample $\sim 2 L^{*}$ ), and the rms of mass fluctuations (in redshift-distorted space) in a $\Lambda \mathrm{CDM}$ background (see Sect. 6). This value $(\sim 1.07 \pm 0.06)$ is in excellent agreement with what one would independently obtain by combining the linear bias parameter measured by Verde et al. (2002) for the whole $2 \mathrm{dFGRS}(1.04 \pm 0.11)$ with the bias scaling law recipe of Norberg et al. (2001), i.e., $b\left(z=0.17, L=2 L^{*}\right)=$ $1.07 \pm 0.13$. 
We can conclude that the time dependence of biasing is marginal $(\mathrm{d} b / b \sim 7 \pm 25 \%)$ for $z<0.8$ while it is substantial $(\mathrm{d} b / b \sim 33 \pm 18 \%)$ in the resdhift interval [0.8-1.5]. The observed time evolution of bias is well described by the simple scaling relationship $b_{\mathrm{L}}=1+(0.03 \pm 0.01)(1+z)^{3.3 \pm 0.6}$ in the interval $0<z<1.5$.

Assuming a linear biasing scheme, one may note that this result was already implicit in Fig. 7 of Sect. 4 . The rms fluctuations of the mass density field on a $8 \mathrm{~h}^{-1} \mathrm{Mpc}$ scale decrease monotonically with redshift by a factor of $\sim 22 \%$ and $\sim 23 \%$ in the redshift intervals [0.17-0.8] and [0.8-1.4], respectively; thus, a nearly constant bias is predicted in the redshift range $z=[0.17-0.8]$ because the rms fluctuations of the galaxy density field are also decreasing by a factor $\sim 16 \%$ in this same interval. Since, instead, $\sigma_{8}$ of galaxies is marginally increasing in the range $z=0.8-1.4(\mathrm{~d} \sigma / \sigma \sim 10 \%$, see Table 1$)$, over this redshift baseline the biasing evolves rapidly.

vii) Bright galaxies are more biased mass tracers than the general population (see Fig. 12). This result confirms and extends into the high redshift domain the luminosity dependence of biasing which is observed in local samples of galaxies (e.g., Benoist et al. 1996; Giuricin et al 2001; Norberg et al. 2001; Zehavi et al. 2002). Specifically, in Fig. 14 we show the dependence of galaxy biasing from luminosity measured in the redshift interval $0.4<z<0.9$ using three different volumelimited VVDS subsamples (i.e., $\mathcal{M}_{B}-5 \log h<-17.7,<-18.7$ and $<-20$ respectively) and compare their linear biasing parameters with those observed locally for a sample of objects having the same median luminosities of the VVDS subsamples (i.e., $L / L^{*}=0.52,0.82,2.0$ respectively). The local estimates have been computed on the basis of the scaling relationship $b / b^{*}=0.85+0.15 L / L^{*}$ derived by Norberg et al. (2001) using the 2dFGRS sample, assuming the $b^{*}$ value given by Verde et al. (2002). As shown above for the volume-limited sample, no significant evolution is seen up to $z \sim 0.8$ also when the dependence of bias from luminosity is analyzed.

Finally, we note that, as already discussed in Sect. 5, galaxies with the same luminosity at different redshifts may actually correspond to different populations. Since, as we have shown, biasing increases with luminosity also at high redshift, and since the measured value of $\mathcal{M}^{*}$ for our sample at redshift $z=0.4(1.5)$ (Paper II) is fainter(brighter) than the cut-off magnitude $\mathcal{M}_{B}^{c}=-20+5 \log h$, we can infer that $b_{\mathrm{L}}(z)$ for a population of objects selected, at any given redshift, in a narrow luminosity range around $\mathcal{M} *(z)$ should increase with redshift even more than what we have measured for our volume-limited sample (see Fig. 13). A more detailed analysis of the biasing for $\mathcal{M}^{*}(z)$ galaxies will be presented in the future, when a larger VVDS data sample will be available.

\subsubsection{Biasing as a function of galaxy color}

Results summarized in Table 3 and presented in Fig. 15 show that, on scales $R=8 h^{-1} \mathrm{Mpc}$, the red sample is a more biased tracer of mass than the blue one in every redshift interval. Similarly to what we have found for the global population, there is some indication of a systematic increase as a function
Table 3. The biasing parameters for red and blue VVDS subsamples on a scale $R=8 h^{-1} \mathrm{Mpc}$.

\begin{tabular}{lllll}
\hline \hline $\begin{array}{l}\text { Redshift } \\
\text { range }\end{array}$ & $\begin{array}{l}\text { Volume } \\
\text { limited }\end{array}$ & $b_{\mathrm{L}}^{\text {rel }}$ & $b_{\mathrm{L}}^{R}$ & $b_{\mathrm{L}}^{B}$ \\
\hline $0.7<z<0.9$ & No & $1.3 \pm 0.2$ & $1.3 \pm 0.5$ & $1.0 \pm 0.5$ \\
$0.9<z<1.1$ & No & $1.3 \pm 0.2$ & $1.4 \pm 0.5$ & $1.1 \pm 0.4$ \\
$1.1<z<1.3$ & No & $1.4 \pm 0.2$ & $1.6 \pm 0.5$ & $1.2 \pm 0.4$ \\
$1.3<z<1.5$ & No & $1.4 \pm 0.3$ & $2.3 \pm 0.4$ & $1.6 \pm 0.4$ \\
\hline $0.7<z<0.9$ & -20 & $1.4 \pm 0.3$ & $1.5 \pm 0.6$ & $1.1 \pm 0.6$ \\
$0.9<z<1.1$ & -20 & $1.3 \pm 0.3$ & $1.6 \pm 0.6$ & $1.2 \pm 0.5$ \\
$1.1<z<1.3$ & -20 & $1.5 \pm 0.2$ & $2.0 \pm 0.5$ & $1.3 \pm 0.4$ \\
$1.3<z<1.5$ & -20 & $1.3 \pm 0.3$ & $2.1 \pm 0.4$ & $1.6 \pm 0.4$ \\
\hline
\end{tabular}

of redshift of the biasing of bright red and blue objects even if, because of the large errorbars, this trend is not statistically significant.

We can compare our results to the biasing measured for extremely red objects (EROS), i.e., objects with extremely red colors $\left((R-K)_{\text {vega }}>5\right)$. Using the results of the correlation analysis of Firth et al. (2002), we obtain, for their $(I-H)_{\text {vega }}>3, H_{\text {vega }}<20.5$ sample (which has a median blue luminosity $\left.\mathcal{M}_{B}=-20.3+5 \log h\right), b_{\mathrm{L}}^{\mathrm{EROS}}(z \sim 1.2) \sim 2.3 \pm 0.6$. Considering the results of Daddi et al. (2001), who analyzed a sample of EROS with $(R-K)_{\text {vega }}>5$ (which roughly corresponds to $\left.(I-H)_{\text {vega }}>3\right), K_{\text {vega }}<19.2$ sample, we conclude that $b_{\mathrm{L}}^{\mathrm{EROS}}(z \sim 1.2) \sim 4 \pm 1$. These values for the galaxy biasing are respectively $\sim 0.5$ and $1.8 \sigma$ higher than that measured for our sample of bright $\left(\mathcal{M}_{B}<-20+5 \log h\right)$ but moderately red galaxies $\left(b_{\mathrm{L}}^{r}(z \sim 1.2)=2 \pm 0.5\right)$. One may interpret this results as an indication for the reddest objects being more strongly biased then moderately red galaxies of similar luminsity. Anyway, given the large errorbars, the evidence that, at $z \sim 1.2$, the biasing properties of these two differently selected populations are different is not statistically significant. As a matter of fact, the values quoted above are also consistent with an alternative hypothesis, i.e., the strength of the EROS fluctuations with respect to the mass fluctuations is not exceptional when compared to the density fluctuations observed in a sample of high redshift, moderately red galaxies of similar luminosity.

The specific values of the biasing parameter at each cosmic epoch are affected by large errors due to the sparseness of our volume-limited subsamples, and to the presence of cosmic variance. One way to bypass uncertainties due to cosmic variance consists in computing the relative biasing function $b^{\text {rel }}(\delta)=b^{r}(\delta) / b^{b}(\delta)$ between the red and blue subsamples. As the subsamples are drawn from the same volume, this ratio should be minimally affected by the finiteness of the volume probed by the first epoch VVDS data.

Results about the relative biasing between galaxy of different colors are graphically shown in the lower panel of Fig. 15, while estimates of the corresponding $b_{\mathrm{L}}$ are quoted in Table 3 .

We do not observe any trend in the relative biasing between red and blue volume-limited subsamples in the redshift range $0.7<z<1.5$. Moreover, our best estimate $b_{\mathrm{L}}^{\text {rel }} \sim 1.4 \pm 0.1$ is in excellent agreement with what is found for nearly the same color-selected populations both locally (Willmer et al. (1998) 


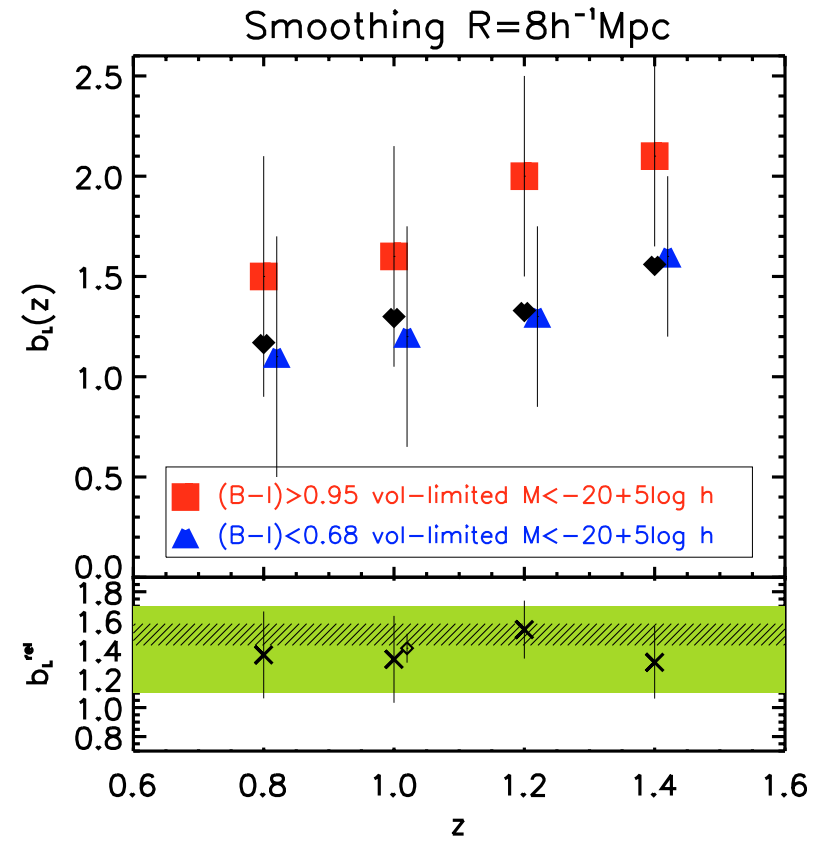

Fig. 15. Upper panel: redshift evolution of the galaxy bias on a scale $R=8 h^{-1} \mathrm{Mpc}$ for the red (squares) and blue (triangles) galaxies in the volume limited samples. For clarity, the triangles have been slightly displaced rightward to avoid crowding. Black diamonds represent the global bias for galaxies brighter than $\mathcal{M}_{B}^{c}=-20+5 \log h$. Lower panel: the relative bias between the red and blue population $\left(b^{\text {rel }}=b^{r} / b^{b}\right)$ is shown as a function of redshift.The filled and shaded areas represents the $1 \sigma$ confidence region of the $z \sim 0$ value for the relative bias derived by Wilmer et al. (1998) and Wild et al. (2005) respectively. The diamond represents the relative bias measured by Coil et al. (2004) in the redshift interval (0.7-1.35).

found that, on a scale $R=8 h^{-1} \mathrm{Mpc}, b_{\mathrm{L}}^{\text {rel }} \equiv b\left((B-R)_{0, \text { vega }}>\right.$ $1.3) / b\left((B-R)_{0, \text { vega }}<1.3\right)=1.4 \pm 0.3$, while Wild et al. (2005) using the $2 \mathrm{dFGRS}$ found on the same scale $b_{\mathrm{L}}^{\text {rel }} \equiv$ $\left.b\left((B-R)_{0, \text { vega }}>1.07\right) / b\left((B-R)_{0, \text { vega }}<1.07\right)=1.5 \pm 0.07\right)$ and at $z \sim 1$ (Coil et al. (2004) found, on a scale $R=8 h^{-1} \mathrm{Mpc}$, that $\left.b_{\mathrm{L}}^{r e l} \equiv b\left((B-R)_{0}>0.7\right) / b\left((B-R)_{0}<0.7\right)=1.41 \pm 0.10\right)$. Thus, VVDS results suggest that there is no-redshift dependence for the relative biasing between red and blue objects up to $z \sim 1.5$. Possible systematics could conspire to produce the observed results; the linear approximation may not always captures, in an accurate way, all the information contained in the biasing function, and more importantly, a purely magnitude limited survey samples the red and blue populations at high redshift with a different efficiency (see discussion in Sect. 7.1).

In principle, the relative bias could be further studied as a function of scale. For example, locally, there is evidence of scale dependence in the relative bias with the bias decreasing as scale increases (Willmer et al. 1998, Madgwick et al. 2003, Wild et al. 2005). However, the sample currently available is not sufficiently large to obtain proper statistics on this effect, although this should be measurable from the final data set.

Finally, we note that no differences in the value of $b_{\mathrm{L}}^{\text {rel }}$ are seen by comparing volume-limited subsamples with the fluxlimited one in different redshift intervals (see Table 3).

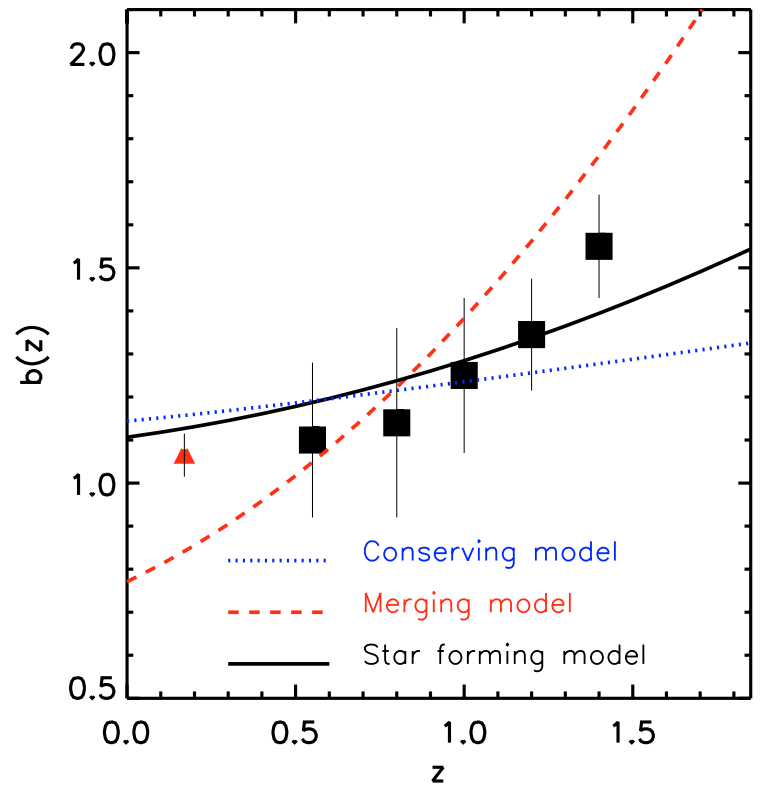

Fig. 16. The redshift evolution of the linear biasing parameter $b_{\mathrm{L}}$ for the volume-limited $\left(\mathcal{M}_{B}^{c}=-20+5 \log h\right)$ sample (see Fig. 13) is compared to various theoretical models of biasing evolution. The dotted line indicates the conserving model normalized at $b_{f}(z=1.4)=1.28$, the solid and dashed lines represent the star forming and merging models with the mass thresholds set at $3.2 \times 10^{11}$ and $2.4 \times 10^{12} h^{-1} M_{\odot}$ respectively.

Thus, we can deduce that in each redshift bins $b^{R}\left(\mathcal{M}_{B}<\right.$ $-20) / b^{R} \sim b^{B}\left(\mathcal{M}_{B}<-20\right) / b^{B}$. In other terms the biasing between the most luminous objects of a particular color and the global population of objects of the same type appears to be independent of galaxy colors (see Table 3 ).

\section{Comparison with theoretical predictions}

In this section we compare our results about the biasing of the $\mathcal{M}_{B}^{c}=-20+5 \log h$ volume-limited, global galaxy sample, with predictions of different theoretical models.

Since we have found that the distribution of galaxy and mass fluctuations are different and the bias was systematically stronger in the past, we can immediately exclude the scenario in which galaxies trace the mass at all cosmic epochs. We thus consider more complex theoretical descriptions of the biasing functions, in particular three different pictures based on orthogonal ideas of how evolution proceeds: the conserving, the merging, and the star forming biasing models (see e.g., Moscardini et al. 1998).

In the first model the number of galaxies is conserved as a function of time (Dekel \& Rees 1987; Fry 1996). This model does not assume anything about the distribution and mass of dark matter halos or their connection with galaxies. In this scheme one assumes that galaxies are biased at birth and then they follow the flow of matter without merging, in other terms they behave as test particles dragged around by the surrounding density fluctuations. Because the acceleration on galaxies is the same as that on the dark matter, the gravitational evolution after formation will tend to bring the bias closer to unity, as described by Fry (1996) and Tegmark \& Peebles (1998). 
Table 4. Best fitting parameters and the corresponding $\chi^{2}$ values for various biasing models.

\begin{tabular}{lll}
\hline \hline Model & Best fitting parameters & $\chi^{2} /$ d.o.f. \\
\hline Conserving & $b_{f}(z=1.4)=1.28 \pm 0.03$ & 2 \\
Merging & $M=2.4 \times 10^{12} h^{-1} M_{\odot}$ & 5.5 \\
Star forming & $M=(3.2 \pm 3) \times 10^{10} h^{-1} M_{\odot}$ & 0.7 \\
\hline
\end{tabular}

The evolution of the bias is given by (e.g., Tegmark \& Peebles 1998)

$b(z)=1+\left(b_{\mathrm{f}}-1\right) \frac{D\left(z_{\mathrm{f}}\right)}{D(z)}$

where $b_{\mathrm{f}}$ is the bias at the formation time $z_{\mathrm{f}}$.

An alternative picture for the bias evolution, which explicitly takes into account galaxy merging, has been proposed by Mo \& White (1996) who gave analytical prescriptions for computing the bias of halos using the Press \& Schechter formalism.

If we explicitly assume that galaxies can be identified with dark matter halos, an approximate expression for the biasing of all halos of mass $>M$ existing at redshift $z$ (but which collapsed at redshift greater than the observation redshift, see discussion in Matarrese et al. 1997) is given by

$b(M, z)=1+\frac{1}{\delta_{\mathrm{c}}}\left(\frac{\delta_{c}^{2}}{\sigma^{2}(M, z)}-1\right)$

where $\delta_{\mathrm{c}} \sim 1.69$ is the linear overdensity of a sphere which collapses in an Einstein-de Sitter Universe and $\sigma(M, z)$ is the linear rms fluctuations on scales corresponding to mass $M$ at the redshift of observation.

The third model is also framed within the peaks-biasing formalism. It assumes that the distribution of galaxies with luminosity $>L$ is well traced by halos with mass $>M$, and predicts the biasing of objects that just collapsed at the redshift of observation (e.g., Blanton et al. 2000). In this star forming model,

$b(M, z)=1+\frac{\delta_{\mathrm{c}}}{\sigma^{2}(M, z)}$

represents the biasing of galaxies that formed in a narrow time interval around redshift $z$ (i.e., galaxies which experienced recent star formation at redshift $z$ ).

Clearly the above models, are based on a set of theoretical ingredients which represent a crude approximation of the complex multiplicity of physical phenomena entering the cosmic recipe of galaxy biasing. In this context, our goal is to investigate the robustness of the simplifying assumptions on which theoretical models are based, and explore the validity or limits of their underlying physical motivations.

Theoretical predictions are compared to observations (VVDS data plus the local normalization derived from 2dFGRS data) in Fig. 16. The best fitting parameters for each model are evaluated using a $\chi^{2}$ statistics and are quoted, together with the corresponding minimum $\chi^{2}$ value of the fit, in Table 4 .

The best fitting galaxy conserving model is obtained when the bias at birth is $b_{\mathrm{f}}\left(z_{\mathrm{f}}=1.4\right)=1.28 \pm 0.03$ and the corresponding normalized $\chi^{2}$-value is $\chi_{N}^{2}=2$. As shown in Fig. 16 the redshift evolution predicted by this model is much weaker than suggested by data. Thus, the gravitational debiasing is a physical mechanism that alone may not fully explain the observed redshift evolution of the biasing, in the sense that it significantly underpredicts the rate of evolution.

The redshift evolution is more pronounced in the merging model (specifically, in Fig. 16, we show the bias evolution of galaxies hosted in halos having mass $M \gtrsim 2.4 \times 10^{12} h^{-1} M_{\odot}$ ). While this model successfully describes the redshift dependence of the biasing of halos (Mo \& White 1996; Somerville et al. 2001) it poorly accounts for the redshift evolution of the bias of galaxies (with $\mathcal{M}_{B} \leq-20+5 \log h$ ) between $z=0$ and $z=1.5$ which is slower than predicted $\left(\chi_{N}^{2}=5.5\right.$ for the best fitting model). Thus, although merging is an important mechanism for describing the evolution of matter clustering, our result implies that merging processes affect galaxies in a less dramatic way than halos. Since in the Press \& Schechter formalism halos are required to merge instantaneously in bigger units at the redshift of observation, our result would imply, also, that the merger time-scales of galaxies is different from that of halos. Moreover, selecting galaxies with a fixed luminosity threshold may not correspond, over such a wide $z$ range as that investigated here, to selecting halos above a given fixed mass threshold. In this sense our result would be suggestive of evolution in the mass-to-light ratio as a function of time.

In Fig. 16 we also show the expected redshift evolution for the star forming model for halos of $M \gtrsim 3.2 \times 10^{10} h^{-1} M_{\odot}$. In this case, the agreement between model and observations is better $\left(\chi_{N}^{2}=0.7\right)$. Clearly this does not mean that we are analyzing a sample of objects that just collapsed and formed stars at the time they were observed; as a matter of fact the model cannot capture all the physical processes shaping the biasing relation. Moreover, the low value fitted for the mass threshold is somewhat unrealistic for the bright objects we are considering. Notwithstanding, Blanton et al. (2000) already noted that the prediction of this biasing model is not much different from the biasing evolution expected for the general population of galaxies in a hydrodynamical simulation of the large scale structure.

Our analysis seems to suggest the apparent need of more complex biasing models that better approximate the observed biasing evolution. Understanding our results completely, however, will require more discriminatory power in the data, and, thus, a larger VVDS sample.

\section{Summary and conclusions}

Deep surveys of the Universe provide the basic ingredients needed to compute the probability distribution function of galaxy fluctuations and to constrain its evolution with cosmic time. The evolution of the galaxy PDF may shed light onto the general assumption that structures grows via gravitational collapse of density fluctuations that are small at early times. When this statistic is combined with analytical CDM predictions for the PDF of mass, useful insights into the biasing function relating mass and galaxy distributions can be obtained.

In this paper, we have explored the potentiality of this approach by analyzing the first-epoch data of the VVDS survey. This is the largest, purely flux-limited sample of 
spectroscopically measured galaxies, currently available in a continuously connected volume and with a robust sampling up to redshifts $z \sim 1.5$. The VVDS is probing the high redshift domain at $I \leq 24$ in the VVDS-02h-4 field with the same sampling rate of pioneer surveys of the local Universe such as the CFA (at $z \sim 0$ ) and, more recently, the $2 \mathrm{dFGRS}$ (at $z \sim 0.1$ ).

Particular attention has been paid to assess the completeness of the VVDS sample and to test the statistical reliability of the PDF of VVDS galaxy fluctuations. In particular:

a) by applying the VVDS observational selection functions to GALICS semi-analytical galaxy simulations we have explored the region of the parameter space where the PDF of VVDS-like densities traces in a statistically unbiased way the parent underlying PDF of the real distribution of galaxy overdensities.

b) we have reconstructed the VVDS galaxy density field on different scales $R=[5,8,10] h^{-1} \mathrm{Mpc}$ by correcting the density estimator for various VVDS selection functions. The final density map for the flux-limited sample has been Wiener filtered in order to minimize the shot-noise contribution.

By studying the PDF of galaxies in the high redshift Universe we have found that the peak of the galaxy PDF systematically shifts to lower density contrasts as a function of redshift and that the probability of observing underdense regions is greater at $z \sim 0.7$ than it was at $z \sim 1.5$. Both these effects provide strong supporting evidence for the standard assumption that the large scale structure is the result of the gravitational growth of small primordial density fluctuations in an expanding universe.

First, within the paradigm of gravitational instability, the assembling process of the large-scale structures is thought to be regulated by the interplay of two competing effects: the tendency of local self-gravity to make overdense regions collapse and the opposite tendency of global cosmological expansion to move them apart. A key signature of gravitational evolution of density fluctuations in an expanding Universe is that underdense regions, experiencing the cosmological matter outflow, occupy a larger volume fraction at present epoch than in the early Universe. Secondly, both these effects, the peak shift and the development of a low density tail, could indicate the existence of a time-evolving biasing between matter and galaxies, since galaxy biasing, systematically increasing with redshift, offers a natural mechanism to re-map the galaxy PDF into progressively higher intervals of density contrasts.

This last interpretation is confirmed by our measurements of the evolution properties of the second and third moments of the galaxy PDF. We find that i) the rms amplitude of the fluctuations of bright VVDS galaxies is with good approximation constant over the full redshift baseline investigated. Specifically we have shown that, in redshift space, $\sigma_{8}$ for galaxies brighter than $\mathcal{M}_{B}^{\mathrm{c}}=-20+5 \log h$ has a mean value of $0.94 \pm 0.07$ in the redshift interval $0.7<z<1.5$; ii) the third moment of the PDF, i.e., the skewness, increases with cosmic time. Its value at $z \sim 1.5$ is nearly $2 \sigma$ lower than measured locally by the 2dFGRS. Both these results, when compared to predictions of linear and second order perturbation theory, unambiguously indicate that galaxy biasing is an increasing function of redshift.
Exploiting the sensitivity of the galaxy PDF to the specific form of the mass-galaxy mapping, we have derived the redshift-, density-, and scale-dependent biasing function $b(z, \delta, R)$ between galaxy and matter fluctuations in a $\Lambda \mathrm{CDM}$ Universe, by analyzing the Jacobian transformation between their respective PDFs. Particular attention has been paid to devise an optimal strategy so that the comparison of the PDFs of mass and galaxies can be carried out in an objective and accurate way. Specifically, we have corrected the lognormal approximation, which describes the mass density PDF, in order to take into account redshift distortions induced by galaxy peculiar velocities at early cosmic epochs where the mapping between redshifts and comoving positions is not linear. In this way, theoretical predictions can be directly compared to observational quantities derived in redshift space.

Without a priori parameterizing the form of the biasing function, we have shown its general non trivial shape, and studied its evolution as a function of cosmic epoch. Our main results about biasing in the high redshift Universe can be summarized as follows:

i) we detect non-linear effects in the biasing relation. The ratio between the quadratic and linear term of the biasing expansion (cf. Eq. (35)) is different from zero at a confidence level greater than $3 \sigma$ in all the redshift bins and for all the smoothing scales probed. This result confirms a general prediction of CDM-based hierarchical models of galaxy formation (e.g., Sigad et al. 2000; Somerville et al. 2001). Such non-linear distortions of the biasing function are not observed locally in the 2dFGRS sample, although indirect evidence of a non-linear bias at $z \sim 0$ exists (Benoist et al. 1999, Baugh et al. 2004).

ii) The biasing function rises sharply in underdense regions (the local slope is $b(\delta)>1$ ) indicating that below some finite mass density threshold the formation efficiency of galaxies brighter than $\mathcal{M}_{B}<-20+5 \log h$ drops to zero. This threshold shifts towards higher values of the mass density field as the luminosity or the redshift of the galaxy population increases.

iii) We do not observe the imprints of scale-dependency in the biasing function a behavior in agreement with results derived from more local surveys at $z \sim 0$ (Verde et al. 2002).

iv) By representing the biasing function in linear approximation, we have found that the linear biasing parameter $b_{\mathrm{L}}$ evolves with cosmic time: it appears that we live in a special epoch in which the galaxy distribution traces the underlying mass distribution on large scales $\left(b_{\mathrm{L}} \sim 1\right)$, while, in the past, the two fields were progressively dissimilar and the relative biasing systematically higher. The difference between the value of $b_{\mathrm{L}}$ at redshift $z \sim 1.5$ and $z \sim 0$ for a population of galaxies with luminosity $\mathcal{M}_{B}<-20+5 \log h$ is significant at a confidence level greater than $3 \sigma\left(\Delta b_{\mathrm{L}} \sim 0.5 \pm 0.14\right)$. In this interval, the essential characteristics of the time evolution of the linear bias are well described in terms of the phenomenological relationship $b_{\mathrm{L}}=1+(0.03 \pm 0.01)(1+z)^{3.3+0.6}$.

v) Over the redshift baseline investigated, the rate of biasing evolution is a function of redshift: $z \sim 0.8$ is the 
characteristic redshift which marks the transition from a "minimum-evolution" late epoch to an early period where the biasing evolution for a population of $\mathcal{M}_{B}<-20+$ $5 \log h$ galaxies is substantial $(\sim 33 \pm 18 \%$ between redshift 0.8 and 1.5$)$.

vi) Brighter galaxies are more strongly biased than less luminous ones at every redshift and the dependence of biasing on luminosity at $z \sim 0.8$ is in good agreement with what is observed in the local Universe.

vii) By comparing our results to predictions of theoretical models for the biasing evolution, we have shown that the galaxy conserving model (Fry 1996) and halo merging (Mo \& White 1996) model offer a poor description of our data. This result could suggest that the gravitational debiasing and the hierarchical merging of halos may not be the only physical mechanisms driving the evolution of galaxy biasing across cosmic epochs. At variance with these results, the star forming model (Blanton et al. 2000) seems to describe better the observed redshift evolution of the linear biasing factor.

viii) After splitting the first-epoch redshift catalog into red and blue volume-limited subsamples, we have found that the red sample is systematically a more biased tracer of mass than the blue one in every redshift interval investigated, but the relative biasing between the two populations is nearly constant in the redshift range $0.7<z<1.5\left(b^{r} / b^{b} \sim\right.$ $1.4 \pm 0.1)$, and comparable with local estimates. Moreover, we have found that the bright red subsample is biased with respect to the general red population in the same way as the bright sample of blue objects is biased with respect to the global blue population thus indicating that biasing as a function of luminosity might be, at first order, independent of color.

ix) Because the VVDS and various EROS samples are not yet large enough, the bias of our sample of bright and moderately red objects at $z \sim 1$ is not statistically dissimilar from that expected for EROS of similar luminosity, even if the EROS biasing appears to be systematically larger.

One key aspect of this paper is the measure of evolution in the distribution properties of galaxy overdensities from a continuous volume sampled with the same selection function over a wide redshift baseline. As our volume sampled is still limited, errors in the analysis presented in this paper are dominated by cosmic variance. The technique presented here will be applied to a larger sample as the VVDS observational program progresses.

Acknowledgements. We would like to acknowledge useful discussions with A. Dekel, R. Giovanelli and L. Moscardini. We also thank S. Andreon and J. Afonso for their useful comments on the paper. This research has been developed within the framework of the VVDS consortium and it has been partially supported by the CNRSINSU and its Programme National de Cosmologie (France), and by the Italian Ministry (MIUR) grants COFIN2000 (MM02037133) and COFIN2003 (num.2003020150). CM also acknowledges financial support from the Region PACA. The VLT-VIMOS observations have been carried out on guaranteed time (GTO) allocated by the European Southern Observatory (ESO) to the VIRMOS consortium, under a contractual agreement between the Centre National de la Recherche Scientifique of France, heading a consortium of French and Italian institutes, and ESO, to design, manufacture and test the VIMOS instrument. We thank the GALICS group for privileged access to their semi-analytic simulations. The mass simulations used in this paper were carried out by the Virgo Supercomputing Consortium using computers based at the Computing Centre of the Max-Planck Society in Garching and at the Edinburgh parallel Computing Centre.

\section{References}

Adelberger, K. L., Steidel, C. C., Giavalisco, M., et al. 1998, ApJ, 505, 18

Arnouts, S., Cristiani, S., Moscardini, L., et al. E. 1999, MNRAS, 310, 540

Bagla, J. S. 1998, MNRAS, 299, 417

Basilakos, S., \& Plionis, M. 2001, MNRAS, 316, 779

Bardeen, J., Bond, J. R., Kaiser, N., \& Szalay, A. 1986, ApJ, 304, 15

Baugh, C. M., Croton, D. J., Gastañaga, E., et al. 2004, MNRAS, 351, L44

Benoist, C., Maurogordato, S., da Costa, L. N., Cappi, A., \& Schaeffer, R. 1996, ApJ, 472, 452

Benoist, C., Cappi, A., da Costa, L. N., et al. 1999, ApJ, 514, 563

Bernardeau, F., Colombi S., Gaztanaga E., \& Scoccimarro, R. 2002, Phys. Rep. 367, 1

Bernardeau, F. 1994, ApJ, 433, 1

Bernardeau, F., \& Kofman, L. 1995, ApJ, 443, 479

Blaizot, J., Wadadekar, Y., Guiderdoni, B., et al. 2003, [arXiv: astro-ph/0309305]

Blanton, M., Cen, R., Ostriker, J. P., \& Strauss, M. A. 1999, ApJ, 522, 590

Blanton, M., Cen, R., Ostriker, J. P., Strauss, M. A., \& Tegmark, M., 2000, ApJ, 531, 1

Bottini et al. (the VVDS team), 2005, PASP, in press, [astro-ph/0409252]

Branchini, E., Freudling, W., Da Costa, L. N., et al. 2001, MNRAS, 326,1191

Carlberg, R. G., Yee, H. K. C., Morris, S. L., et al. 2000, ApJ, 542, 57

Catelan, P., Matarrese, S., \& Porciani, C. 1998, ApJ, 502, L1

Cen, R., \& Ostriker, J. P. 1992, ApJ, 399, L113

Coil, A., Davis, M., Madgwick, D. S., et al. 2004, ApJ, 609, 525

Colberg, J. M., White, S. D. M., Yoshida, N., et al. (The Virgo Consortium), 2000, MNRAS, 319, 209

Coles, P., \& Jones, B. 1991, MNRAS, 248, 1

Coles, P. 1993, MNRAS, 262, 1065

Colless, M. M., Dalton, G., Maddox, S., et al. 2001, MNRAS, 328, 1039

Croton, D. J. 2004, MNRAS, 352, 1232

Daddi, E., Broadhurst, T., Zamorani, G., et al. 2001, A\&A, 376, 825

Dalal, N., \& Kochanek, C. S. 2002, ApJ, 572, 25

Davis, M., \& Geller, M. J. 1976, ApJ, 254, 437

Davis, M., \& Huchra, J. 1981, ApJ, 254, 437

Davis, M., \& Djorgovski, S. 1985, ApJ, 299, 15

Davis, M., Efstathiou, G., Frenk, C., \& White, S. D. M. 1985, ApJ, 292, 371

Davis, M., Faber, S. M., Newman, J. et al. 2003, Proc. SPIE, 4834, 161

Dekel, A. 1994, ARA\&A, 32, 371

Dekel, A., \& Lahav, O. 1999, ApJ, 520, 24

Dekel, A., \& Rees, M. J. 1987, Nature, 326, 455

Dressler, A. 1980, ApJ, 236, 351

Evrard, A. E., Summers, F. J., \& Davis, M. 1994, ApJ, 422, 11 
Firth, A. E., Somerville, R. S., McMahon, R. G. et al. 2002, MNRAS, 332,617

Fisher, K. B., Davis, M., Strauss, M. A., Yahil, A., \& Huchra, J. P. 1993, ApJ, 402, 42

Foucaud, S., McCracken, H. J., Le Fèvre, O., et al. 2003, A\&A, 409, 835

Fry, J. N. 1985, ApJ, 289, 10

Fry, J. N., \& Gaztañaga, E. 1993, ApJ, 413, 447

Fry, J. N. 1996, ApJ, 461, L65

Giavalisco, M., Steidel, C. C., Adelberger, K. L., et al. 1998, ApJ, 503, 543

Giovanelli, R., Haynes, M., \& Chincarini G. 1986, ApJ, 300, 77

Giovanelli, R., Haynes, M. P., Freudling, W., et al. 1998, ApJ, 505, 91

Giuricin, G., Samurovic, S., Girardi, M., Mezzetti, M., \& Marinoni, C. 2001, ApJ, 554, 857

Guzzo, L., Strauss, M. A., Fisher, K. B., Giovanelli, R., \& Haynes, M. P. 1997, ApJ, 489, 37

Hamilton, A. J. S. 2001, MNRAS, 322, 419

Hamilton, A. J. S. 1988, ApJ, 331, L59

Heat, D. J. 1977, MNRAS, 179, 351

Hatton, S., Deviendrt, J. E. G., Ninin, S., et al. 2003, MNRAS, 373 , 75

Hudson, M. 1993, MNRAS, 265, 43

Hui, L., Gaztañaga, E. 1999, ApJ, 519, 622

Ilbert, O., et al. (the VVDS team) 2005, A\&A, 439, 863 (Paper II)

Juszkiewicz, R., Bouchet, F. R., \& Colombi, S. 1993, ApJ, 412, L9

Kaiser, N. 1984, ApJ, 284, L9

Kaiser, N. 1987, MNRAS, 227, 1

Kaiser, N., \& Peacock, J. A. 1991, ApJ, 379, 482

Kayo, I., Taruya, A., \& Suto, Y. 2001, ApJ, 561, 22

Klypin, A., Kravtsov, A. V., Valenzuela, O., \& Prada, F. 1999, ApJ, 522,82

Kofman, L., Bertshinger, E., Gelb, J. M., Nusser, A., \& Dekel, A. 1994, ApJ, 420, 44

Kravtsov, A. V., \& Klypin A. A. 1999, ApJ, 520, 437

Lahav, O., Itoh, M., Inagaki, S., \& Suto, Y. 1993, ApJ, 402, 387

Lahav, O., Fisher, K. B., Hoffman, Y., Scharf, C. A., \& Zaroubi, S. 1994, ApJ, 423, L93

Lahav, O., Bridle, S. L., Percival, W. J. et al. 2002, MNRAS, 333, 961

Lahav, O., Lilje, P. B., Primack, J. R., Rees, M. J. et al. 1991, MNRAS, 251,128

Le Fèvre, O., Hudon, D., Lilly, S. J., et al. 1996, ApJ, 461, 534

Le Fèvre, O., Vettolani, G., Maccagni, D., et al. (the VVDS team) 2003, The Messenger, 111, 18

Le Fèvre, O., Vettolani, G., Paltani, S. et al. (the VVDS team) 2004, A\&A, 428, 1043

Le Fèvre, O., et al. (the VVDS team) 2005a A\&A, 439, 845 (Paper I)

Le Fèvre, O., et al. (the VVDS team) 2005b, A\&A, 439, 877 (Paper III)

Le Fèvre, O., et al. (the VVDS team) 2005c, Nature, in press

Madgwick, D. S.,Lahav, O., Baldry, I. K. et al. 2002, MNRAS, 333, 133

Magliocchetti, M., Bagla, J. S., Maddox, S., \& Lahav, O. 2000, MNRAS, 314, 546
Mann, R. G., Peacock, J. A., \& Heavens, A. F. 1998, MNRAS, 293, 209

Marinoni, C., Monaco, P., Giuricin, G., \& Costantini, B. 1998, ApJ, 505,484

Marinoni, C., \& Hudson, M. J. 2002, ApJ, 569, 101

Martel, H. 1991, ApJ, 377, 7

Matarrese, S., Coles, P., Lucchin, F., \& Moscardini, L. 1997, MNRAS, 286, 115

Maurogordato, S., \& Lachièze-Rey, M. 1987, ApJ, 320, 13

Mc Cracken, H., Radovich, M., Bertin, E., et al. 2003, A\&A, 410, 17

Mo, H., \& White, S. D. M. 1996, MNRAS, 282, 347

Moore, B. 1999, ApJ, 524, L19

Moscardini, L., Coles, P., Lucchin, F., \& Matarrese, S. 1998, MNRAS, 299, 95

Navarro, J. F., Frenk C. S., \& White S. D. M. 1997, ApJ, 490, 493

Norberg, P., Baugh, C. M., Hawkins, E., et al. 2001, MNRAS, 328, 64

Oke, J. B., \& Gunn, J. E. 1983, ApJ, 266, 713

Ostriker, J. P., Nagamine, K., Cen, R., \& Fukugita, M. 2003, ApJ, 597, 1

Peebles, P. J. E. 1980, The Large Scale Structure of the Universe (Princeton: Princeton Univ Press)

Pollo, A., et al. (the VVDS team) 2005, A\&A, 439, 887

Press, W. H., Teukolsky, S. A., Vetterling, W. T., \& Flannery, B. P. 1992, Numerical Recipes, (Cambridge: University Press)

Rybicki, G. B., \& Press, W. H. 1992, ApJ, 398, 169

Saslaw, W. C., Gravitational Physics of Stellar and Galactic Systems (Cambridge: Cambridge Univ. Press)

Sigad, Y., Branchini, E., \& Dekel, A. 2000, ApJ, 540, 62

Small, T. A., Ma, C., Sargent, W. L. W., \& Hamilton, D. 1999, ApJ, 524,31

Somerville, R. S., Lemson, G., Sigad, Y., et al. 2001, MNRAS, 320, 289.

Steidel, C. C., Adelberger, K. L., Dickinson, M. E., et al. 1998, ApJ, 492, 428

Strauss, M. A., \& Willick, J. A. 1995, Phys. Rev. 261, 271

Szapudi, I., \& Pan, J. 2004, ApJ, 602, 26

Taruya, A., \& Suto, Y. 2000, ApJ, 542, 559

Taylor, A. N., \& Watts, P. I. R. 2000, MNRAS, 314, 92

Tegmark, M., \& Peebles, P. J. E. 1998, ApJ, 500, L79

Ueda, H., \& Yokoyama, J. 1996, MNRAS, 280, 754

Verde, L., Heavens, A. F., Percival, W. J., et al. 2002, MNRAS, 335, 432

Weinberg, D. H., Davé, R., Katz, N., \& Hernquist, L. 2004, ApJ, 601, 1

White, S. D. M., \& Rees, M. J. 1978, MNRAS, 183, 341

White, S. D. M., Tully, B. R., \& Davis, M. 1988, ApJ, 333, L45

Wild, V., Peacock, J. A., Lahav, O., et al. 2005, MNRAS, 356, 247

Willmer, C. N. A, da Costa, L, N., \& Pellegrini, P. S. 1998, AJ, 115, 869

Zaroubi, S., Hoffman, Y., Fisher, K. B., \& Lahav, O. 1995 ApJ, 449, 446

Zehavi, I. Blanton, M. R., Frioeman, J. A. et al. 2002, ApJ, 571, 172

Zucca, E. et al. (the VVDS team), 2005, A\&A, submitted, [arXiv: astro-ph/0506393] 
C. Marinoni et al.: The VVDS: Galaxy biasing up to $z=1.5$, Online Material $p 1$

\section{Online Material}




\section{Appendix A}

Here we describe the application of the Wiener filtering technique to deconvolve the noise signature from the VVDS density map. We de-noise data in Fourier space, noting, however, that an equivalent filtering can be directly applied in real space (e.g., Rybicki \& Press 1992; Zaroubi et al. 1995).

Let us assume that the observed smoothed density field $\delta_{\mathrm{O}}(\boldsymbol{x})$, and the true underlying density field $\delta_{\mathrm{T}}(\boldsymbol{x})$, smoothed on the same scale, are related via

$\delta_{\mathrm{O}}(\boldsymbol{x})=\delta_{\mathrm{T}}(\boldsymbol{x})+\epsilon(\boldsymbol{x})$,

where $\epsilon(\boldsymbol{x})$ is the local contribution from shot noise (see Eq. (8)). The Wiener filtered density field, in Fourier space, is

$\tilde{\delta}_{\mathrm{F}}(\boldsymbol{k})=\mathcal{F}(\boldsymbol{k}) \tilde{\delta}_{\mathrm{O}}(\boldsymbol{k})$,

$\mathcal{F}(\boldsymbol{k})=\frac{\left\langle\tilde{\delta}_{\mathrm{T}}^{2}(\boldsymbol{k})\right\rangle}{\left\langle\tilde{\delta}_{\mathrm{T}}^{2}(\boldsymbol{k})\right\rangle+(2 \pi)^{3} P_{\epsilon}(\boldsymbol{k})}$

where brackets denote statistical averages and where $P_{\epsilon}(\boldsymbol{k})=$ $(2 \pi)^{-3}\left\langle\left|\tilde{\epsilon}^{2}(\boldsymbol{k})\right|\right\rangle$ is the power spectrum of the noise. Assuming ergodic conditions for the noise, we can derive its power spectrum as $P_{\epsilon}(\boldsymbol{k})=(2 \pi)^{-3}|\tilde{\epsilon}(\boldsymbol{k})|^{2}$.

The Power spectrum of the underlying theoretical density distribution of galaxies, smoothed with the window $F$ and taking into account the VVDS geometrical constraints, can be derived from Eq. (5). Specifically, the theoretical overdensity field smoothed on a certain scale $R$, which is sampled by an idealized survey with no selection functions, is

$\delta_{\mathrm{g}}(\boldsymbol{r}, R) \equiv \frac{1}{\bar{\rho}} \sum_{p} \delta^{D}\left(\boldsymbol{r}-\boldsymbol{r}_{p}\right) * F\left(\frac{\left|\boldsymbol{r}-r_{p}\right|}{R}\right)-1$.

If we assume that this density field is periodic on same volume $V$, having, for example, the same geometry of the VVDS survey, its Fourier transform is

$\tilde{\delta}_{\mathrm{T}}(\boldsymbol{k})=\frac{1}{\bar{\rho}} \sum_{i} \frac{n_{i}}{V} \mathrm{e}^{\mathrm{i} \boldsymbol{k} \cdot \boldsymbol{r}_{i}} \tilde{F}_{k}-\frac{1}{V} \int \mathrm{e}^{\mathrm{i} \boldsymbol{k} \cdot \boldsymbol{r}} \mathrm{d}^{3} \boldsymbol{r}$

where the mean theoretical galaxy density $\bar{\rho}$ may be estimated averaging over sufficiently large volumes the VVDS data corrected for selection functions and sampling rate (see Eq. (6)).

In Eq. (44), $n_{i}$ represent the occupation numbers of the infinitesimal cells $\mathrm{d} \boldsymbol{r}_{i}$ in which the VVDS volume can be partitioned $\left(n_{i}=0\right.$ or 1$)$ and the sum is intended over all the cells of the survey volume. The Fourier transform of the smoothing window function $F$ with which the discontinuous galaxy density field is regularized is, in the case of a Top-Hat spherical smoothing filter,

$\tilde{F}_{k}=3\left[\frac{\sin (k R)}{(k R)^{3}}-\frac{\cos (k R)}{(k R)^{2}}\right]$.

In order to obtain an estimate of the quantity $\left\langle\tilde{\delta}_{\mathrm{T}}^{2}(\boldsymbol{k})\right\rangle$ that enters the Wiener filter definition (Eq. (42)), we compute the ensemble average of the squares of the modulus of the Fourier amplitudes (Eq. (44)) and obtain

$$
\begin{aligned}
\left\langle\tilde{\delta}_{\mathrm{T}}(\boldsymbol{k}) \tilde{\delta}_{\mathrm{T}}^{*}\left(\boldsymbol{k}^{\prime}\right)\right\rangle= & \frac{1}{(\bar{\rho} V)^{2}}\left[\sum_{i} \sum_{j}\left\langle n_{i} n_{j}\right\rangle \mathrm{e}^{\mathrm{i}\left(\boldsymbol{k} \cdot \boldsymbol{r}_{i}-\boldsymbol{k}^{\prime} \cdot \boldsymbol{r}_{j}\right)} \tilde{F}_{k} \tilde{F}_{k^{\prime}}^{*}\right. \\
+ & W_{k} W_{k^{\prime}}^{*}-\bar{\rho} V \sum_{i}\left\langle n_{i}\right\rangle \mathrm{e}^{\mathrm{i} \boldsymbol{k} \cdot \boldsymbol{r}_{i}} \tilde{F}_{k} W_{k^{\prime}}^{*} \\
& \left.-\bar{\rho} V \sum_{j}\left\langle n_{j}\right\rangle \mathrm{e}^{\mathrm{i} \boldsymbol{k}^{\prime} \cdot \boldsymbol{r}_{j}} \tilde{F}_{k^{\prime}}^{*} W_{k}\right]
\end{aligned}
$$

where

$W(\boldsymbol{k})=\frac{1}{V} \int \mathrm{e}^{\mathrm{i} \boldsymbol{k} \cdot \boldsymbol{r}} \mathrm{d}^{3} \boldsymbol{r}$.

The mean value of the occupation number is given by (Peebles 1980)

$\left\langle n_{i} n_{j}\right\rangle=\bar{\rho}^{2} \mathrm{~d}^{3} \boldsymbol{r}_{i} \mathrm{~d}^{3} \boldsymbol{r}_{j}\left[1+\xi\left(\boldsymbol{r}_{i}-\boldsymbol{r}_{j}\right)\right]$

$\left\langle n_{i}\right\rangle^{2}=\left\langle n_{i}\right\rangle=\bar{\rho} \mathrm{d}^{3} \boldsymbol{r}_{i}$

where the correlation function may be expressed via the Fourier conjugates

$\xi\left(\boldsymbol{r}_{i j}\right)=\frac{1}{(2 \pi)^{3}} \int \mathrm{d}^{3} \boldsymbol{k} P(\boldsymbol{k}) \mathrm{e}^{-\mathrm{i} \boldsymbol{k} \cdot \boldsymbol{r}_{i j}}$

$P(\boldsymbol{k})=\int \mathrm{d}^{3} \boldsymbol{r} \xi(\boldsymbol{r}) \mathrm{e}^{\mathrm{i} \boldsymbol{k} \cdot \boldsymbol{r}}$

We convert the sums into integrals over the occupation cells taking into account the specific VVDS geometry and obtain

$\begin{aligned}\left\langle\tilde{\delta}_{\mathrm{T}}^{2}(\boldsymbol{k})\right\rangle= & \left|\tilde{F}_{k}\right|^{2} \int d^{3} \boldsymbol{k}^{\prime} P\left(\boldsymbol{k}^{\prime}\right) G\left(\boldsymbol{k}-\boldsymbol{k}^{\prime}\right) \\ & +\left|\tilde{F}_{k}^{2}\right|(\bar{\rho} V)^{-1}+\left|W_{k}\right|^{2}\left(1-\tilde{F}_{k}\right)^{2}\end{aligned}$

were

$G\left(\boldsymbol{k}-\boldsymbol{k}^{\prime}\right)=\frac{1}{(2 \pi)^{3}}\left|W\left(\boldsymbol{k}-\boldsymbol{k}^{\prime}\right)\right|^{2}$.

Note that, in the idealized conditions of a survey of infinite spatial extension $(V \rightarrow \infty)$ and no smoothing applied to data the previous expression reduces to

$\left\langle\tilde{\delta}_{\mathrm{T}}^{2}(\boldsymbol{k})\right\rangle=(2 \pi)^{3} P\left(\boldsymbol{k}^{\prime}\right) \delta^{D}\left(\boldsymbol{k}-\boldsymbol{k}^{\prime}\right)$.

Given the quasi pencil-beam nature of the first season VVDS data, however, the sample of the $\delta$ field can be described, to a good approximation, as confined to a cylinder of length $L$ (aligned along the redshift direction) and radius $R$. In this case the Fourier transform of the survey window function (cf. Eq. (46)) is given by

$W_{k}=j_{0}\left(\frac{L}{2} k_{\|}\right) 2 \frac{J_{1}\left(k_{\perp} R\right)}{k_{\perp} R}$

where $j_{0}$ and $J_{1}$ are the spherical and first kind Bessel functions and where $k=\sqrt{k_{\|}^{2}+k_{\perp}^{2}}$.

The convolution integral on the right hand side of Eq. (50) can then be evaluated as follows (e.g., Kaiser \& Peacock 1991; Fisher et al. 1993)

$\int \mathrm{d}^{3} \boldsymbol{k}^{\prime} P\left(\boldsymbol{k}^{\prime}\right) G\left(\boldsymbol{k}-\boldsymbol{k}^{\prime}\right)=\frac{2}{\pi V} \int_{-\infty}^{\infty} \mathrm{d} y j_{0}^{2}\left(y-k \frac{L}{2}\right) F(y)$ 


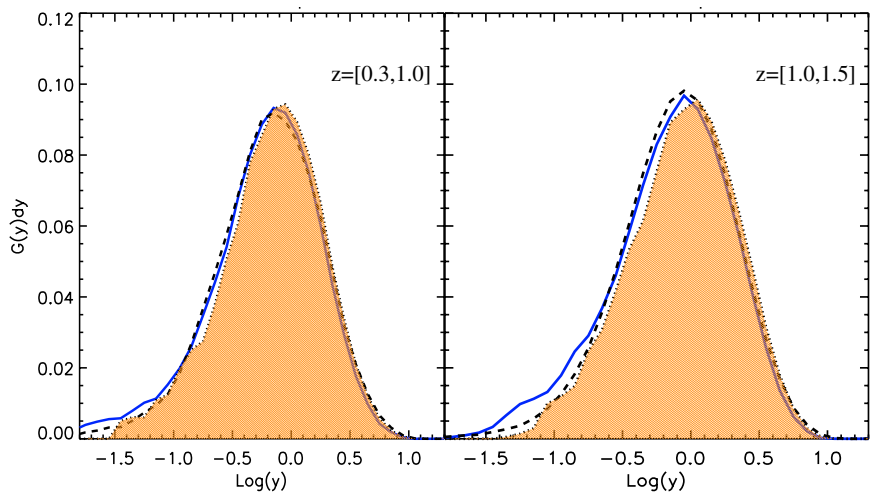

Fig. A.1. The overdensity distribution reconstructed on a scale $R=$ $8 h^{-1} \mathrm{Mpc}$ and in two different redshift intervals using the GtALICS semi-analytical simulation (see Sect. 4.1) is plotted as a function of $\log \left(1+\delta_{\mathrm{g}}\right)$. The dashed line represent the distribution of overdensities traced by simulated galaxies with $\mathcal{M}<-15+5 \log h$. The shaded area (dotted line) represents the observed overdensity distribution i.e., the distribution recovered after applying to the simulation the VVDS flux limit $(I \leq 24)$ and all the underlying VVDS instrumental selection effects (see Sect. 4.1). The solid line represent the distribution of density contrasts after correcting the observed distribution with the Wiener technique.

where $V=\pi R^{2} L$ is the volume of the cylinder and where

$F(x)=\int_{0}^{\infty} P\left(\frac{\sqrt{x^{2}+x^{\prime 2}}}{R}\right) \frac{J_{1}^{2}\left(x^{\prime}\right)}{x^{\prime}} \mathrm{d} x^{\prime}$.

Note that the theoretically expected variance of the density field in the VVDS volume is derived in real comoving space. Thus, we have corrected the theoretical predictions (cf. Eq. (50)) in order to take into account redshift space distortions induced by galaxy peculiar velocities (see discussion in Sect. 5).
We compute the galaxy density field on a regular Cartesian grid of spacing $0.5 \mathrm{~h}^{-1} \mathrm{Mpc}$ using the smoothing scheme presented in Eq. (5). The resulting 3D density map is then Fourier transformed in redshift slices having line-of-sight dimensions $\mathrm{d} z=0.1$. This partition strategy is implemented in order to describe consistently, using cylindrical approximations, the deep survey volume of the VVDS (whose comoving transversal dimensions are an increasing function of distance.) The Wiener filter at each wave-vector position is then computed by using Eq. (50). Finally, as described in Sect. 3, we select only the Wiener filtered density fluctuations recovered in spheres having at least $70 \%$ of their volume in the 4-passes, VVDS-02h-4 field.

In Fig. A.1 we use the GALICS semi-analytical simulation (see Sect. 4.1), to show the effect of the Wiener filter on the reconstructed galaxy density field. As Fig. A.1 shows, the net effect of the correction is to shift towards low-values the density contrasts having low signal-to-noise ratio (by definition $F$ is always smaller than unity). Figure A.1 shows that i) at the same density, the effects of the correction are bigger at high redshift where the density field is noisier due to the increasing sparseness of a flux-limited sample, and ii) at the same redshift the Wiener filter mostly affects the low-density tail of the distribution where the counts within the TH window are small. It is also evident from Fig. A.1 that, in the density interval $-1<\log \left(1+\delta_{\mathrm{g}}\right)<1$, the Wiener filtered distribution offers a better approximation of the underlying PDF, than the observed (uncorrected) overdensity distribution. 